\title{
A moment projection method for population balance dynamics with a shrinkage term
}

\author{
Shaohua $\mathrm{Wu}^{\mathrm{a}}$, Edward K. Y. Yapp ${ }^{\mathrm{b}}$, Jethro Akroyd ${ }^{\mathrm{b}}$, Sebastian Mosbach ${ }^{\mathrm{b}}$, \\ Rong $\mathrm{Xu}^{\mathrm{c}}$, Wenming Yang ${ }^{\mathrm{a}}$, Markus Kraft*b,c \\ ${ }^{a}$ Department of Mechanical Engineering, National University of Singapore, \\ Engineering Block EA, Engineering Drive 1, Singapore, 117576 \\ ${ }^{b}$ Department of Chemical Engineering and Biotechnology, University of Cambridge, \\ New Museums Site, Pembroke Street, Cambridge, CB2 3RA United Kingdom \\ ${ }^{c}$ School of Chemical and Biomedical Engineering, Nanyang Technological University, \\ 62 Nanyang Drive, Singapore, 637459 \\ corresponding author* \\ E-mail:mk306@cam.ac.uk
}

\begin{abstract}
A new method of moments for solving the population balance equation is developed and presented. The moment projection method (MPM) is numerically simple and easy to implement and attempts to address the challenge of particle shrinkage due to processes such as oxidation, evaporation or dissolution. It directly solves the moment transport equation for the moments and tracks the number of the smallest particles using the algorithm by Blumstein and Wheeler [Phys. Rev. B, 8:1764-1776, 1973]. The performance of the new method is measured against the method of moments (MOM) and the hybrid method of moments (HMOM). The results suggest that MPM performs much better than MOM and HMOM where shrinkage is dominant. The new method predicts mean quantities which are almost as accurate as a high-precision stochastic method calculated using the established direct simulation algorithm (DSA).
\end{abstract}


Keywords: method of moments, shrinkage, oxidation, population balance equation

\section{Introduction}

Population balance equations (PBEs) have received considerable interest 3 in the chemical engineering field due to its wide ranging applications from 4 soot formation in combustion [1] to crystallisation [2]. The PBE describes the evolution of a particle size distribution (PSD) that is dependent on time, 6 spatial location and a set of internal coordinates which characterise particle 7 properties (e.g., surface area, volume and chemical composition) [3-6]. A 8 typical PBE contains an inception term describing the formation of particles

from the surrounding fluid, a coagulation term due to the collision and sticking of particles, a growth term due to surface reaction and condensation on individual particles, and a shrinkage term due to oxidation, evaporation or dissolution. In mathematics, PBEs are a series of integro-differential equations which are often so complex that analytical solutions rarely exist [7].

A number of methods have been proposed to solve these types of equations [8-10]. In ref. [11] a stochastic method is developed to solve the PBE describing the evolution of soot particles in laminar premixed flames. Soot particles are represented by an ensemble of stochastic particles and particle processes are treated probabilistically [11, 12]. The simulations can be proven to converge to the deterministic solution of the PBE [13]. However, the simulations can be prohibitively expensive when extended to particles with multidimensional internal coordinates $[14,15]$. In sectional methods, the PSD is discretised into a number of bins, or sections. The PBE is then 
transformed into a set of ordinary differential equations (ODEs) describing the evolution of quantities such as the mass and number of particles within each bin. Many of the proposed methods are limited to specific grids or to specific forms of the PBE. In ref. [16] a fixed pivot method is developed which is able to evolve any two arbitrary distribution properties by representing the PSD as a delta function within each bin [16-20]. The moving pivot approach [16], which is an extension of the fixed pivot method, takes the pivot as the location of the delta function within each bin. When the PSD is heavily weighted towards one end of some of the bins, the moving pivot approach is more accurate than the fixed pivot approach. Recently, the traditional sectional method $[21,22]$ has been extended to conserve more than two moments in the discretised solution of the PBE using a high-order method [23]. Similar to stochastic methods, sectional methods are intuitive and accurate. However, a large number of bins may be required to obtain good accuracy which can make the method computationally expensive [24].

For PBEs with only one or two internal coordinates the method of moments (MOM) is widely used because of the low computational cost [25-29]. The PBE is multiplied by property functions, e.g., integer powers of the internal coordinates, and integrated over state space. The resulting ODEs are then solved to yield integral quantities such as total particle number and mass. Depending on the coagulation kernel used the moment transport equations may not be closed, i.e., presence of fractional- or negativeorder moments. In general, there are two ways to close the equations: (1) create a functional relationship between unknown moments and transported moments such as in the method of moments with interpolative closure 
(MOMIC) [6, 30, 31]; or (2) reconstruct the PSD from the transported moments and approximate the unclosed terms using Gauss quadrature such as in the quadrature method of moments (QMOM) or direct quadrature method of moments (DQMOM) [24, 28, 29, 32-34]. MOMIC has been widely used due to its numerical simplicity and ease of implementation while being reasonably accurate in dealing with inception, coagulation and growth processes [30]. In ref. [35] it is found that the solution obtained using QMOM and DQMOM showed an excellent agreement with the analytical solution for aggregation and breakage problems. A review of the models of particle formation and the numerical methods used to solve them can be found in ref. [36].

However, MOMIC, QMOM and DQMOM all fail in the treatment of shrinkage problems, where the pointwise value of the PSD at the smallest particle mass is required to close the moment equations [7, 37, 38]. Note that where the term shrinkage is used, it is implied that depletion is included. This problem is addressed in ref. [39] by introducing a source term for the smallest particles in what is known as the hybrid method of moment (HMOM). HMOM adopts the idea of DQMOM where the PSD is discretised into small and large particles and the production of the smallest particle is assumed to be proportional to the mass lost from the large particles. However, as we will show later, this assumption is too coarse and can overestimate the production of the smallest particles. In ref. [37] a finite-size domain complete set of trial functions method of moments (FCMOM) is proposed that uses a series of Legendre polynomials to obtain a continuous reconstruction of the PSD, thus generating information about the smallest particles. However, this approach cannot guarantee the positivity of the reconstructed PSD because 
only a finite number of polynomials can be determined [38]. An alternative method is the extended quadrature method of moments (EQMOM) [38] where the PSD is approximated by continuous non-negative kernel density functions, e.g., gamma, beta or lognormal functions. High accuracy can be achieved in terms of the reconstructed PSD. Information about the shape of the PSD is needed a priori to select a suitable kernel density function; otherwise, a large number of kernel functions are required which can make this method excessively complicated and computationally expensive.

The purpose of this paper is to present a new method, the moment projection method (MPM), which is able to robustly handle the shrinkage of particles while retaining numerical simplicity. The paper is organized as follow. Section 2 presents moment methods for solving the population balance equation. The detailed mathematical formulation of MPM and related algorithms are introduced. In Section 3, MPM is compared with MOM, HMOM and the stochastic method for the processes of inception, coagulation, growth and shrinkage. In Section 4 principal conclusions are summarised.

\section{Moment methods for solving the population balance equation}

\subsection{Population balance equation}

We consider a spatially homogeneous problem with a discrete-mass distribution where the smallest particles have a mass of $m_{1}$ and particles in the mass class $i$ have a mass of $m_{i}=i m_{1}$ [31]. All particles are spherical and have constant density. The PBE governing the evolution of the distribution can be written as: 


$$
\frac{\mathrm{d} N(i, t)}{\mathrm{d} t}=R(i, t)+G(i, t)+W(i, t)+S(i, t), \quad i=1,2, \ldots, \infty,
$$

where $N(i, t)$ is the number of particles belonging to the mass class $i$ at time $t$ (which we will refer to as $N_{i}$ from hereon), and $R, G, W$ and $S$ are the inception, coagulation, surface growth and shrinkage terms, respectively, the notation consistent with ref. [30]. This is known as a particle number representation of the PSD. The specific functional forms of the source terms used in this work will be discussed in Section 3.

\subsection{Moment equation}

As already mentioned before, an efficient approach for solving the PBE is MOM where the PBE is transformed into a set of moment equations and integral values such as the total particle number and mass can be computed. This is achieved by applying the definition, moment of order $k$ of the PSD

$$
M_{k}=\sum_{i=1}^{\infty} i^{k} N_{i}, \quad k=0,1,2, \ldots
$$

to Eq. (1), leading to:

$$
\frac{\mathrm{d} M_{k}}{\mathrm{~d} t}=R_{k}(M)+G_{k}(M)+W_{k}(M)+S_{k}\left(M, N_{1}\right) .
$$

Note that the source terms on the right-hand side of Eq. (3) are now a function of moments; in addition, the shrinkage term is a function of the number of the smallest particle, $N_{1}$. When the source terms contain complex kernels, fractional- or negative-order moments are encountered [26]. Therefore, the mathematical difficulty of MOM lies in obtaining closure for these moment 
source terms using a finite set of moments. This requires either a priori specification of the PSD or a suitable closure scheme. In MOMIC [30], closure is accomplished by Lagrange polynomial interpolation of the logarithm of the whole-order moments whose values are available at each integration step of Eq. (3). By separating interpolation for positive- and negative-order moments, MOMIC shows very high accuracy in the treatment of unimodal PSDs undergoing coagulation and growth and also good accuracy for bimodal PSDs formed from the competition between persistent inception and coagulation [30,31]. Another type of closure scheme uses Gauss quadratures such as in QMOM where the PSD is represented by a weighted summation of Dirac delta functions [32]. The general form of the moment equation in QMOM can be written as:

$$
\frac{\mathrm{d} \widetilde{M}_{k}}{\mathrm{~d} t}=R_{k}\left(w_{j}, i_{j}\right)+G_{k}\left(w_{j}, i_{j}\right)+W_{k}\left(w_{j}, i_{j}\right), \quad j=1, \ldots, N,
$$

where $w_{j}$ and $i_{j}$, respectively, are the weights and abscissas of the delta functions which can be derived from the moments using the product difference (PD) algorithm [40]. $N$ is the number of delta functions. $\widetilde{M}$ is the empirical moment determined from the product of $w_{j}$ and $i_{j}$ and, therefore, is an approximation of $M$ of the true PSD. We use the symbol " " to express approximations of the particle quantities of Eqs. (2) and (3). DQMOM is similar to QMOM except that in DQMOM transport equations for $w_{j}$ and $i_{j}$ are directly solved:

$$
\begin{aligned}
\frac{\mathrm{d} w_{j}}{\mathrm{~d} t} & =R_{k}\left(w_{j}, i_{j}\right)+G_{k}\left(w_{j}, i_{j}\right)+W_{k}\left(w_{j}, i_{j}\right), \\
\frac{\mathrm{d} i_{j}}{\mathrm{~d} t} & =R_{k}\left(w_{j}, i_{j}\right)+G_{k}\left(w_{j}, i_{j}\right)+W_{k}\left(w_{j}, i_{j}\right) .
\end{aligned}
$$


Note the absence of a shrinkage source term as both of these methods are unable to handle shrinkage. Although DQMOM is superior to QMOM in terms of computational efficiency [35], to determine the source terms for $w_{j}$ and $i_{j}$, inversion of a matrix composed of the abscissas is required. When some of the abscissas are not distinct the matrix may exhibit singularity problems, i.e., the rank of the matrix is lower than its dimension, thus making its inversion impossible [34]. This implies that not all of the delta functions are required to represent the PSD. This situation arises, for example, when the PSD is unimodal; all the delta functions would be located at the same position associated with the mode of the distribution. This has been addressed by adding small perturbations to the non-distinct abscissas [34]. Another important case is when the PSD is generated from an inception process; at the first time step $w_{j}$ and $i_{j}$ would be undefined. To overcome this problem "seeds" have been introduced with negligibly small weights and abscissas which did not lead to any discernable difference in the moments [34].

\subsection{Moment projection method}

In MPM, we approximate the true PSD by assuming that all particles are distributed into a finite number of particle mass classes. The $k$-th order empirical moment can then be expressed as:

$$
\widetilde{M}_{k}=\alpha{ }_{1}^{k} \widetilde{N}_{\alpha_{1}}+\sum_{j=2}^{N_{\mathrm{p}}} \alpha_{j}^{k} \widetilde{N}_{\alpha_{j}}
$$

where $\alpha_{j}$ is the particle mass and $\tilde{N}_{\alpha_{j}}$ refers to the number of particles of the mass $\alpha_{j} . N_{\mathrm{p}}$ is the number of particle mass classes and is a user-defined parameter. Mathematically, $\alpha_{j}$ and $\widetilde{N}_{\alpha_{j}}$ can be interpreted as the particle 
number representation of $i_{j}$ and $w_{j}$ in QMOM and DQMOM. MPM uses $\alpha_{j}$ and $\widetilde{N}_{\alpha_{j}}$ as an assumption of the form of the PSD itself, in a similar vein to the fixed pivot method [16]. By construction the number of moments that can be obtained are bounded to $N_{\mathrm{p}}$ because the particle masses and particle number determined in MPM only ensure the first few corresponding moments are equal to those from the true PSD:

$$
\widetilde{M}_{k}=M_{k}, \quad k=0, \ldots, 2 N_{\mathrm{p}}-2 .
$$

From Eq. (3), it follows that:

$$
\frac{\mathrm{d} \widetilde{M}_{k}}{\mathrm{~d} t}=R_{k}(\widetilde{M})+G_{k}(\widetilde{M})+W_{k}(\widetilde{M})+S_{k}\left(\widetilde{M}, N_{1}\right) .
$$

In order to evaluate the boundary flux $\left(N_{1}\right)$ present in the shrinkage term, we fix the first particle mass to be equal to the smallest particle mass of the true PSD: $\alpha_{1}=m_{1}$. Therefore, $\widetilde{N}_{\alpha_{1}}$, the number of particles of the mass $\alpha_{1}$, reflects the number of the smallest particles of the true PSD. The moment transport equations in MPM can then be given as:

$$
\frac{\mathrm{d} \widetilde{M}_{k}}{\mathrm{~d} t}=R_{k}(\widetilde{M})+G_{k}(\widetilde{M})+W_{k}(\widetilde{M})+S_{k}\left(\widetilde{M}, \widetilde{N}_{\alpha_{1}}\right) .
$$

The problem now lies in determining $\alpha_{j}$ and $\widetilde{N}_{\alpha_{j}}$ while ensuring that $\alpha_{1}=m_{1}$ (see Eq. (6)). This can be achieved by using the Blumstein-Wheeler algorithm [41] which was originally applied to the moments of the frequency distribution of harmonic solids. A real symmetric tridiagonal matrix is constructed from a series of recursion coefficients of orthogonal polynomials composed of moments [42, 43]. $\alpha_{j}$ and $\widetilde{N}_{\alpha_{j}}$ can be determined by solving for the eigenvalues and eigenvectors of the matrix. As for the requirement that $\alpha_{1}$ 
be fixed to be equal to $m_{1}$, this can be fulfilled simply by modifying the last recursion coefficient of the tridiagonal matrix using $m_{1}$. The full algorithm can be found in Appendix A. Algorithm 1 describes the numerical procedure of MPM.

There are two important differences between MPM and QMOM. First, the source terms for $\widetilde{M}_{k}$ in MPM are directly evaluated using the moment transport equation. This allows us to take advantage of the accuracy and computational efficiency of MOMIC to handle inception, growth and coagulation, while we close the moment equation for shrinkage by approximating the boundary flux term $N_{1}$ with the number of particles of the smallest mass $\widetilde{N}_{\alpha_{1}}$. By contrast, in QMOM evaluation of integrals of the source terms involve the unknown PSD and is approximated with a Gaussian quadrature [24, 33]. The second difference is the algorithm used to obtain $\alpha_{j}$ and $\widetilde{N}_{\alpha_{j}}$ from the moments in MPM, or weights and abscissas in QMOM. In QMOM this is achieved through the Gordon algorithm [40], in which a moment matrix is constructed according to a "product-difference" recursion relation to obtain the coefficients of a continued fraction. While in MPM we apply the Blumstein-Wheeler algorithm [41] where the derivation is given in terms of orthogonal polynomials which is more straightforward than that given by Gordon [40] in terms of continued fractions. Furthermore, this algorithm can be easily modified to treat the cases in which zero, one or two particle mass classes are fixed. 


\section{Algorithm 1: Moment projection method algorithm.}

Input: Moments of the PSD $M_{k}\left(t_{0}\right)$ for $k=0, \ldots, 2 N_{\mathrm{p}}-2$ or the PSD itself

$N\left(i, t_{0}\right)$ for $i=1, \ldots, i_{\max }\left(i_{\max }\right.$ : the largest particle mass $)$ at initial time $t_{0}$; final time $t_{\mathrm{f}}$.

Output: Empirical moments of the PSD $\widetilde{M}_{k}\left(t_{\mathrm{f}}\right)$ for $k=0, \ldots, 2 N_{\mathrm{p}}-2$ at final time $t_{\mathrm{f}}$ where $N_{\mathrm{p}}$ is the number of particle masses used to approximate the PSD.

Calculate the moments of the true PSD using Eq. (2):

$$
M_{k}\left(t_{0}\right)=\sum_{i=1}^{i_{\max }} i^{k} N\left(i, t_{0}\right), \quad k=0, \ldots, 2 N_{\mathrm{p}}-2
$$

For $\widetilde{M}_{k}=M_{k}$, solve Eq. (6) for $\widetilde{N}_{\alpha_{1}}\left(\alpha_{1}\right.$ is fixed) and $\alpha_{j}$ and $\widetilde{N}_{\alpha_{j}}$ $\left(j=2, \ldots, N_{\mathrm{p}}\right)$ using Algorithm 2:

$$
\widetilde{M}_{k}\left(t_{0}\right)=\alpha_{1}^{k} \widetilde{N}_{\alpha_{1}}\left(t_{0}\right)+\sum_{j=2}^{N_{\mathrm{p}}} \alpha_{j}^{k} \widetilde{N}_{\alpha_{j}}\left(t_{0}\right), \quad k=0, \ldots, 2 N_{\mathrm{p}}-2 .
$$

196

$t \longleftarrow t_{0}, \widetilde{M}_{k}(t) \longleftarrow \widetilde{M}_{k}\left(t_{0}\right)$

while $t<t_{f}$ do

Integrate Eq. (9) over the time interval $\left[t_{i}, t_{i}+h\right]$ (using an ODE solver):

$$
\frac{\mathrm{d} \widetilde{M}_{k}}{\mathrm{~d} t}=R_{k}(\widetilde{M})+G_{k}(\widetilde{M})+W_{k}(\widetilde{M})+S_{k}\left(\widetilde{M}, \widetilde{N}_{\alpha_{1}}\right)
$$

with initial condition:

$$
\left(\begin{array}{c}
\widetilde{M}_{k}\left(t_{i}\right) \\
\widetilde{N}_{\alpha_{1}}\left(t_{i}\right)
\end{array}\right)=\left(\begin{array}{c}
\widetilde{M}_{k, i} \\
\widetilde{N}_{\alpha_{1}, i}
\end{array}\right)
$$

where $R_{k}(\widetilde{M}), G_{k}(\widetilde{M}), W_{k}(\widetilde{M})$ and $S_{k}\left(\widetilde{M}, \widetilde{N}_{\alpha_{1}}\right)$ are given by Eqs. (11), (14), (16) and (18), respectively.

Use Blumstein algorithm to update $\alpha_{j}$ and $\widetilde{N}_{\alpha_{j}}$, and assign solution at $t_{i+1}=t_{i}+h$ :

$$
\left(\begin{array}{c}
\widetilde{M}_{k, i+1} \\
\widetilde{N}_{\alpha_{1}, i+1}
\end{array}\right) \leftarrow\left(\begin{array}{c}
\widetilde{M}_{k}\left(t_{i}+h\right) \\
\widetilde{N}_{\alpha_{1}}\left(t_{i}+h\right)
\end{array}\right)
$$

$L \quad i \longleftarrow i+1$ 


\section{Numerical results}

To assess the performance of MPM, numerical results are compared to those from MOM, HMOM and the stochastic method. We test the method for the individual processes of inception, coagulation, growth and shrinkage, then for all of these processes combined. As the focus of this paper is on MPM's ability to handle shrinkage, we devise a number of test cases where different types of PSDs are supplied as the initial condition and present the errors in the moments relative to a high-precision stochastic solution calculated using the direct simulation algorithm (DSA) [13]. The high-precision solution was obtained using 131,072 stochastic particles and a single run; the remainder of the numerical and model parameters used may be found in Table 1. HMOM was originally developed for bivariate PBEs $[39,44]$. We modify this method to make it applicable for monovariate PBEs. Pertinent details of the method can be found in Appendix B.

Table 1: Numerical and model parameters used for stochastic solution

\begin{tabular}{ll}
\hline Description & Value \\
\hline Number of splits & 100 \\
Time step & $0.001 \mathrm{~s}$ \\
Number of stochastic particles & 131,072 \\
Number of runs & 1 \\
Maximum zeroth moment & $1 \times 10^{18} \# / \mathrm{m}^{3}$ \\
\hline
\end{tabular}

In this work constant kernels are used. The use of more realistic Brownian collision kernels would lead to a closure problem due to fractional- 
and negative-order moments which would appear on the right-hand side of Eq. (9). The way in which MPM is formulated means that these source terms can be closed using MOMIC; however, this introduces an interpolation error. The aim here is to investigate the MPM error in isolation.

\subsection{Pure inception}

Inception is the formation of particles from the surrounding fluid and is a common phenomenon in the chemical engineering field. By definition these particles have the smallest mass $m_{1}$ and is assumed to be equal to 1 . In this work the inception rate is assumed to be:

$$
R(i, t)=I_{m_{1}},
$$

where the inception kernel $I_{m_{1}}=100 \mathrm{~s}^{-1}$. The moment source term due to inception can be derived to be:

$$
R_{k}(M)=m_{1}^{k} I_{m_{1}}, \quad k=0, \ldots, 2 N_{\mathrm{p}}-2 .
$$

It can be seen that the moment source term is only dependant on the smallest particle mass and the inception kernel. Simulations are performed where a log-normal distribution is supplied as the initial condition:

$$
N(i, t=0)=100 \exp \left(-(\log (i)-\log (25))^{2} / 0.05\right), \quad i=1,2, \ldots, 100,
$$

which is shown in Fig. 1 (continuous line). Also shown in Fig. 1 (dotted line) is the PSD computed by solving the master equation after 10 seconds of pure inception. It develops a mode at the smallest particles because only particles with the smallest mass are formed. We now want to see whether MPM is able to capture this increase in the number of the smallest particles. 
The particle masses $\alpha_{j}$ and the corresponding number of particles $\widetilde{N}_{\alpha_{j}}$ from MPM are shown in Fig. 2. Four particles masses $\left(N_{\mathrm{p}}=4\right)$ are used to approximate the PSD. As $\alpha_{1}$ is fixed to be equal to the smallest particle mass, the particle masses remain unchanged. The number of particles of the smallest mass $\widetilde{N}_{\alpha_{1}}$ does indeed increase (linear because of constant rate) while the other $\widetilde{N}_{\alpha_{j}}(j=2,3,4)$ do not change. As a further point of comparison the zeroth and first moments are compared with those from MOM, HMOM and the stochastic method in Fig. 3. All the methods give the same results. The continuous inception of particles leads to a linear increase in the total number and mass of particles, $M_{0}$ and $M_{1}$, respectively.

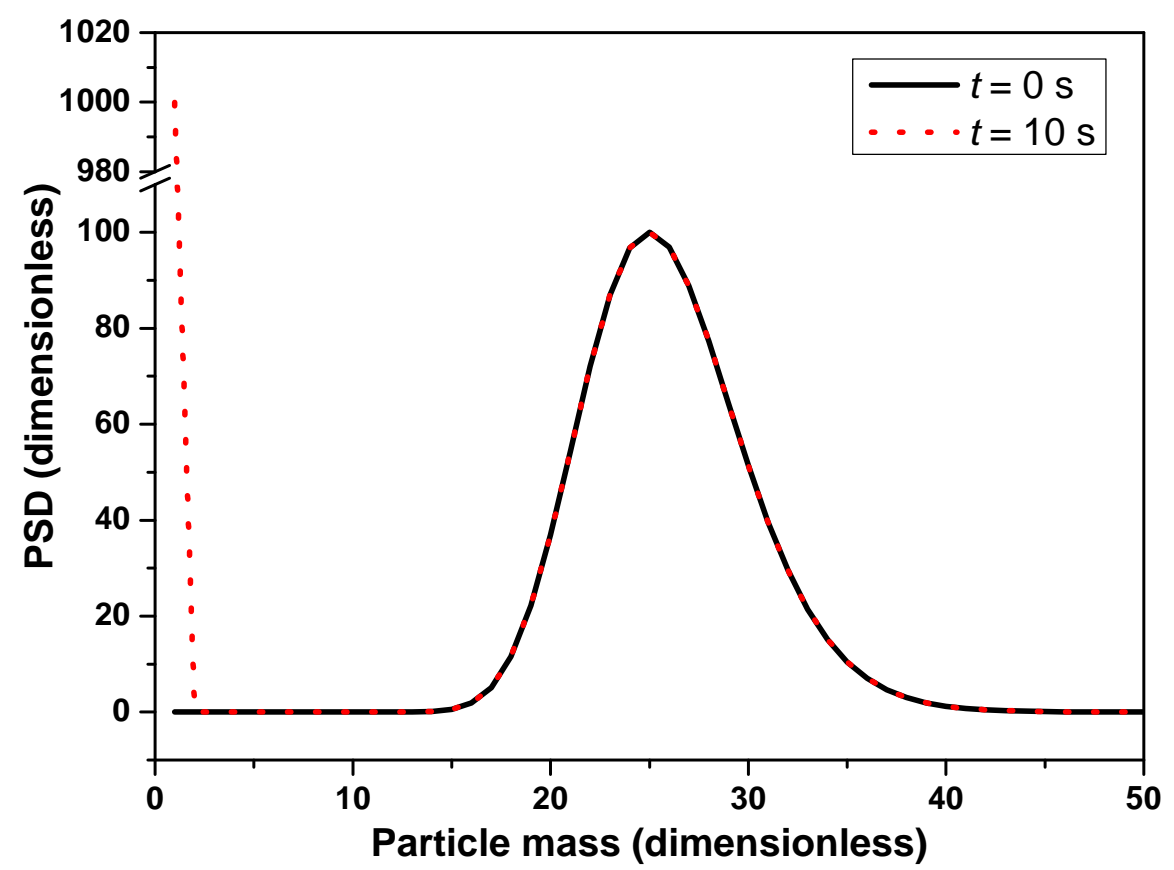

Figure 1: Evolution of the PSD computed by solving the master equation under pure inception. 

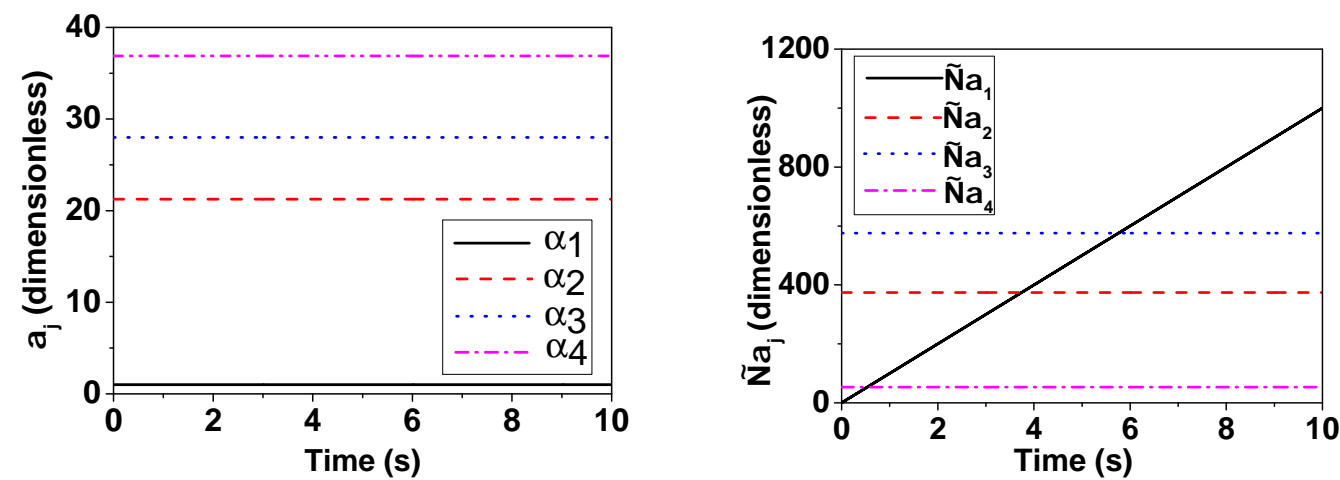

Figure 2: Evolution of the particle masses $\alpha_{j}$ (left panel) and the corresponding number of particles $\widetilde{N}_{\alpha_{j}}$ (right panel) using MPM under pure inception. The PSD at $t=0 \mathrm{~s}$ in Fig. 1 (continuous line) is supplied as the initial condition. A total of four particle masses are used to approximate the PSD.
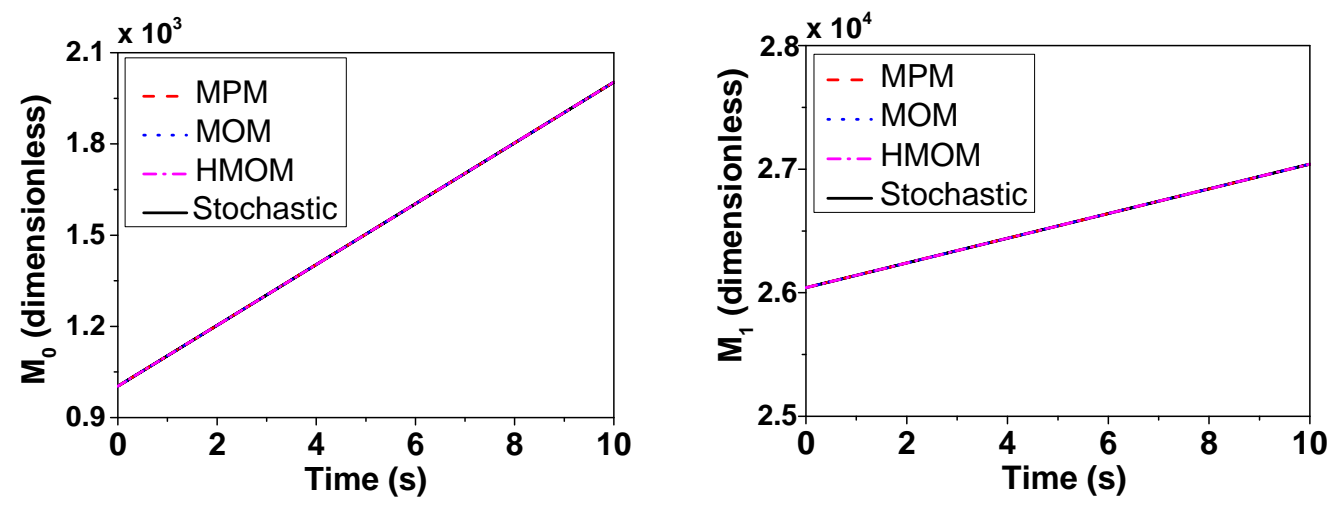

Figure 3: Comparison of the zeroth moment $M_{0}$ (left panel) and the first moment $M_{1}$ (right panel) between MPM, MOM, HMOM and the stochastic method under pure inception.

\subsection{Pure coagulation}

Coagulation is a nonlinear process that describes the collision and sticking of particles. The source term for coagulation considered in this work is of 
the form:

$$
G(i, t)=\frac{1}{2} \sum_{j=1}^{i} K_{\mathrm{Cg}} N_{j} N_{i-j}-\sum_{j=1}^{\infty} K_{\mathrm{Cg}} N_{i} N_{j} .
$$

The first term on the right-hand side of Eq. (13) refers to the formation of particles of mass $i$ due to collisions between all combinations of particles with masses that sum to $i$. It contains a factor of a $1 / 2$ to avoid double counting. The second term represents the destruction of particles of mass $i$ due to collisions between particles of mass $i$ and particles of any other mass $j$. The coagulation kernel $K_{\mathrm{Cg}}$ is usually dependent on the collision regime and the collision diameter. In this work, this kernel is assumed to be a constant: $K_{\mathrm{Cg}}=2 \times 10^{-4} \mathrm{~s}^{-1}$. The moment source term due to coagulation is:

$$
G_{k}(M)= \begin{cases}-1 / 2 K_{\mathrm{Cg}} M_{0}^{2}, & k=0, \\
0, & k=1, \\
\frac{1}{2} \sum_{r=1}^{k-1}\left(\begin{array}{l}
k \\
r
\end{array}\right) K_{\mathrm{Cg}} M_{r} M_{k-r}, & k=2, \ldots, 2 N_{\mathrm{p}}-2 .\end{cases}
$$

The same log-normal distribution in Eq. (12) is supplied as the initial condition and the evolution of the PSD under pure coagulation is shown in Fig. 4. The PSD is computed using the stochastic method because for the given coagulation kernel and simulation time, if the master equation were to be used, particles would rapidly reach the maximum mass class which would introduce errors. Multiple coagulation peaks are formed as particles collide and stick together, and these particles in turn collide and stick, and so forth. Figure 5 shows that $\alpha_{j}(j=2,3,4)$ increase reflecting an increase in the average particle mass. An increase in $\widetilde{N}_{\alpha_{2}}$ is observed at the beginning of the simulation due to the collision and sticking of the smallest particles. The time evolution 
of $M_{0}$ and $M_{1}$ computed using the different methods are compared in Fig. 6. Since no fractional- or negative-order moments are present in the moment source term, all the methods generate the same results. Coagulation is a nonlinear process, therefore, we observe a nonlinear decrease in $M_{0}$ while $M_{1}$ remains unchanged.

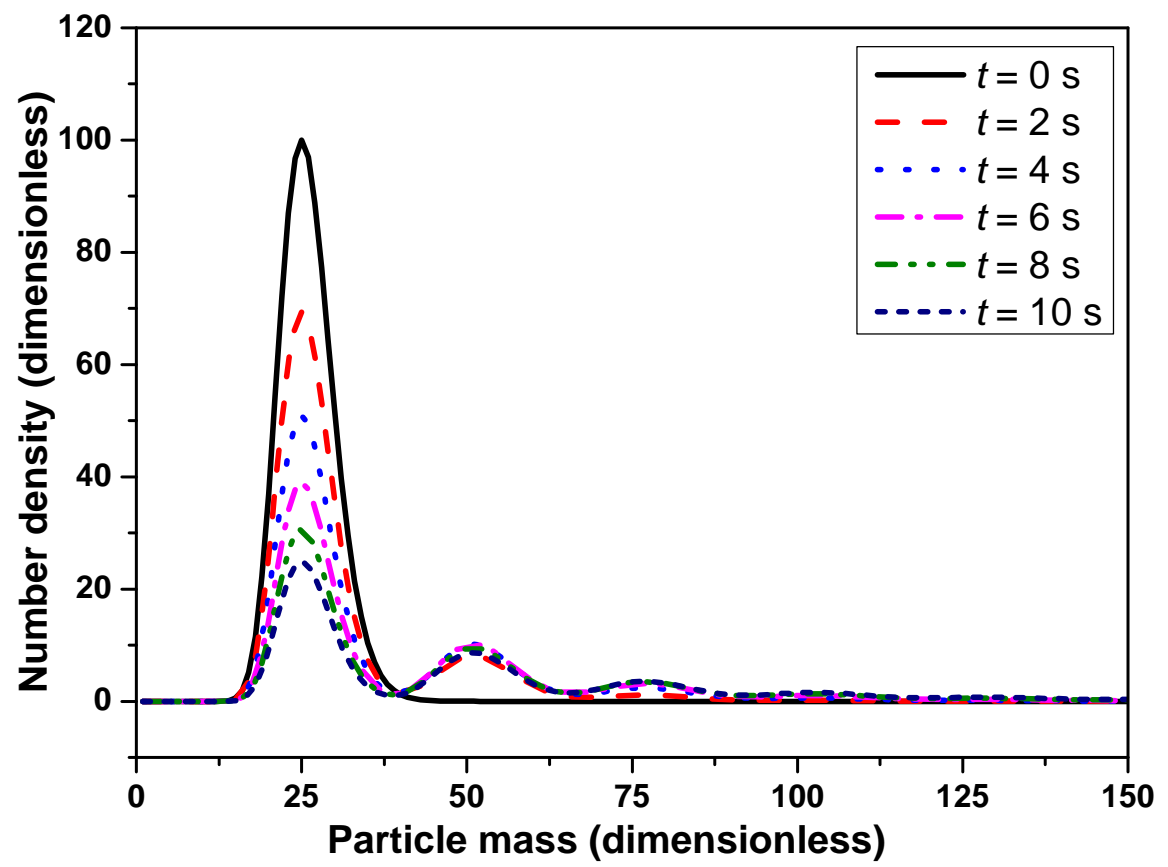

Figure 4: Evolution of the PSD computed using the stochastic method under pure coagulation.

\subsection{Pure growth}

Growth is a process whereby particles increase in mass due to surface reaction or condensation. Here we consider a growth process where its source term is of the form of:

$$
W(i, t)=K_{\mathrm{G}}\left(N_{i-\delta}-N_{i}\right),
$$



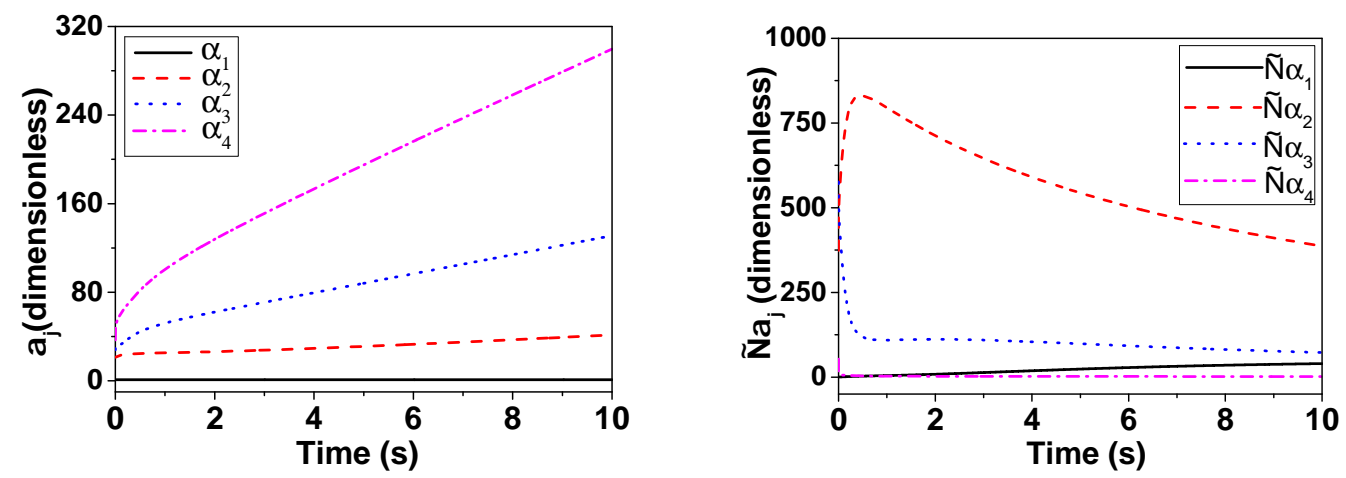

Figure 5: Evolution of the particle masses $\alpha_{j}$ (left panel) and the corresponding number of particles $\tilde{N}_{\alpha_{j}}$ (right panel) using MPM under pure coagulation. The PSD at $t=0 \mathrm{~s}$ in Fig. 4 (continuous line) is supplied as the initial condition. A total of four particle masses are used to approximate the PSD.
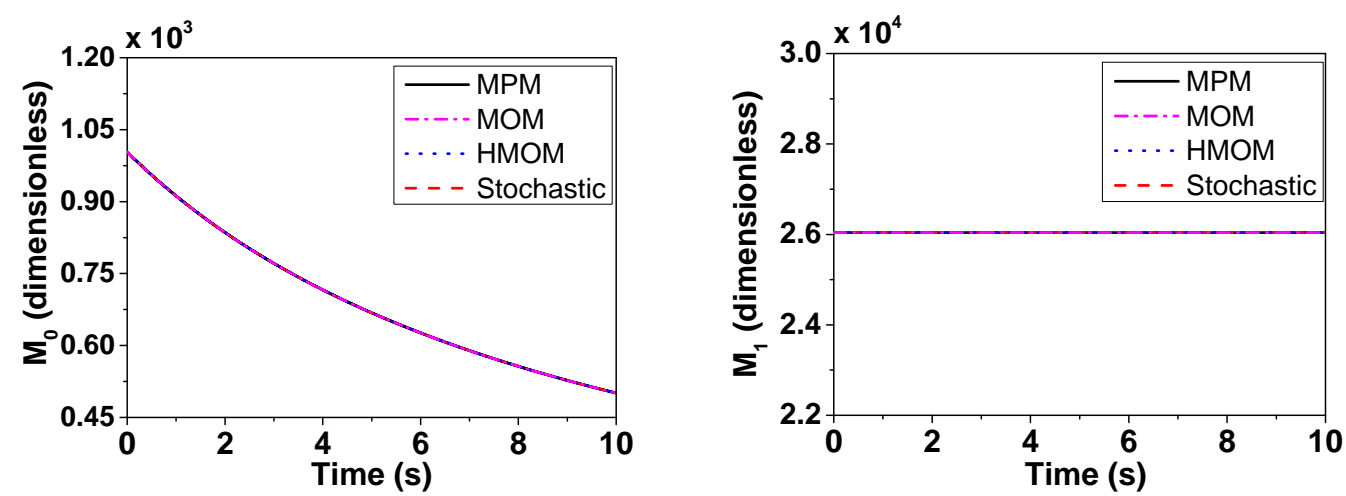

Figure 6: Comparison of the zeroth moment $M_{0}$ (left panel) and the first moment $M_{1}$ (right panel) between MPM, MOM, HMOM and the stochastic method under pure coagulation.

273 where the growth kernel $K_{\mathrm{G}}=20 \mathrm{~s}^{-1}$, and $\delta$ is the change in particle mass 274 after a growth process and is assumed to be 1 . The moment source term can 
275

be expressed as:

$$
W_{k}(M)= \begin{cases}0, & k=0, \\
K_{\mathrm{G}} \sum_{r=1}^{k}\left(\begin{array}{l}
k \\
r
\end{array}\right) \delta^{r} M_{k-r}, & k=1, \ldots, 2 N_{\mathrm{p}}-2 .\end{cases}
$$

Again, the log-normal distribution in Eq. (12) is supplied as the initial condition. Figure 7 shows the evolution of the PSD computed by solving the master equation under pure growth. The PSD shifts towards larger particle masses; however, the distribution widens and the peak decreases in magnitude consistent with a growth process. The simulation results using MPM is similar to that of pure coagulation. $\alpha_{j}(j=2,3,4)$ increase as shown in Fig. 8 and the mean quantities computed using MPM are in agreement with MOM, HMOM and the stochastic method as shown in Fig. 9. The total particle number remains unchanged while a linear increase in the total particle mass is observed.

\subsection{Pure shrinkage}

Shrinkage is the opposite of growth but with an important difference: when particles of the smallest mass shrink they are removed from the system which leads to a decrease in the total particle number. Here we consider the source term for shrinkage of the form:

$$
S(i, t)=K_{\mathrm{Sk}}\left(N_{i+\delta}-N_{i}\right),
$$

where the shrinkage kernel $K_{\mathrm{Sk}}=30 \mathrm{~s}^{-1}$ and $\delta$ is the change in particle mass after a shrinkage process and is assumed to be 1 . The moment source term 


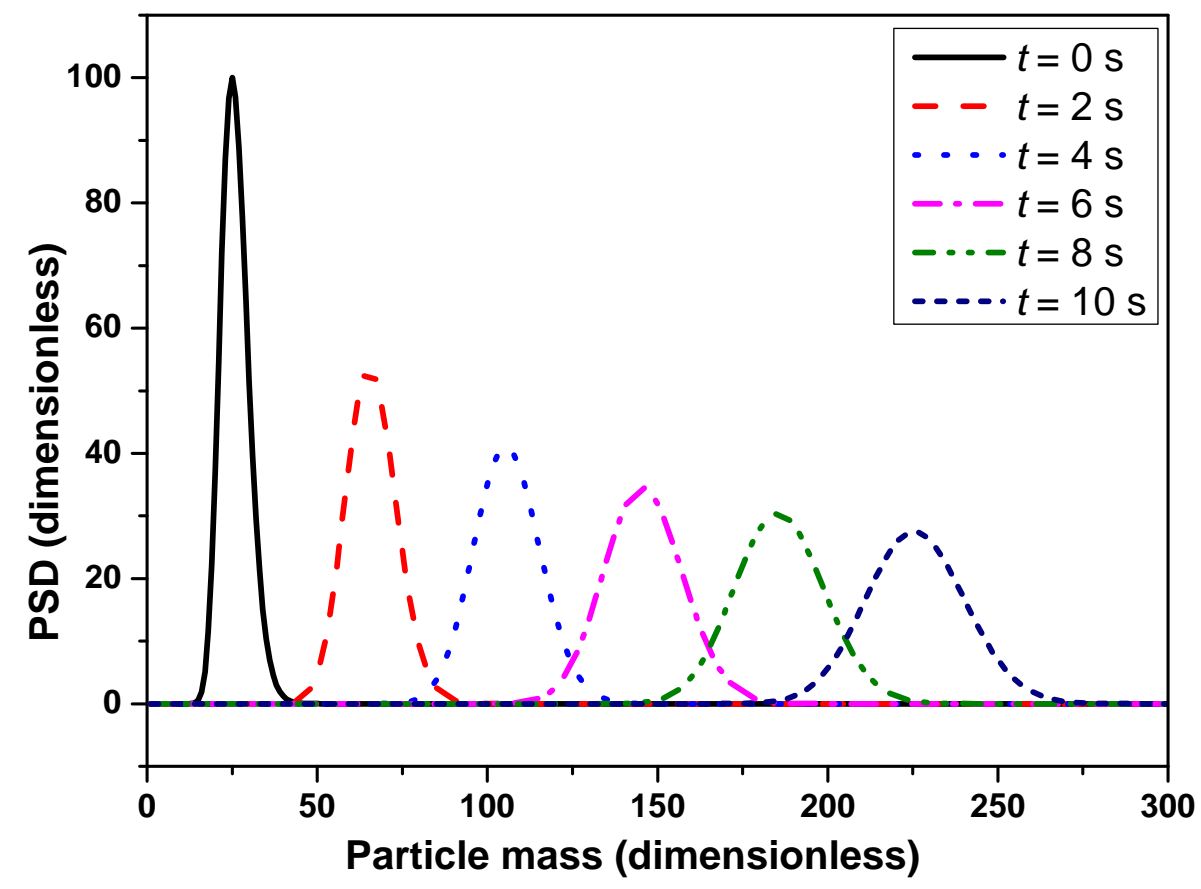

Figure 7: Evolution of the PSD computed by solving the master equation under pure growth.

for shrinkage can then be expressed as:

$$
S_{k}\left(M, N_{1}\right)= \begin{cases}-K_{\mathrm{Sk}} N_{1}, & k=0, \\
K_{\mathrm{Sk}} \sum_{r=1}^{k}\left(\begin{array}{l}
k \\
r
\end{array}\right)(-\delta)^{r} M_{k-r}, & k=1, \ldots, 2 N_{\mathrm{p}}-2 .\end{cases}
$$

294 It can be seen that the zeroth order shrinkage moment source term, $S_{0}$, is dependent on the number of particles of the smallest mass, $N_{1}$. To obtain closure of Eq. (18), $N_{1}$ has to be determined. However this value is unknown because it depends on the number of the larger particles which shrink to form the smallest particles. A worst case scenario is assuming $N_{1}=0$ when solving MOM for shrinkage such as used in this work. In MPM, we fix the first 

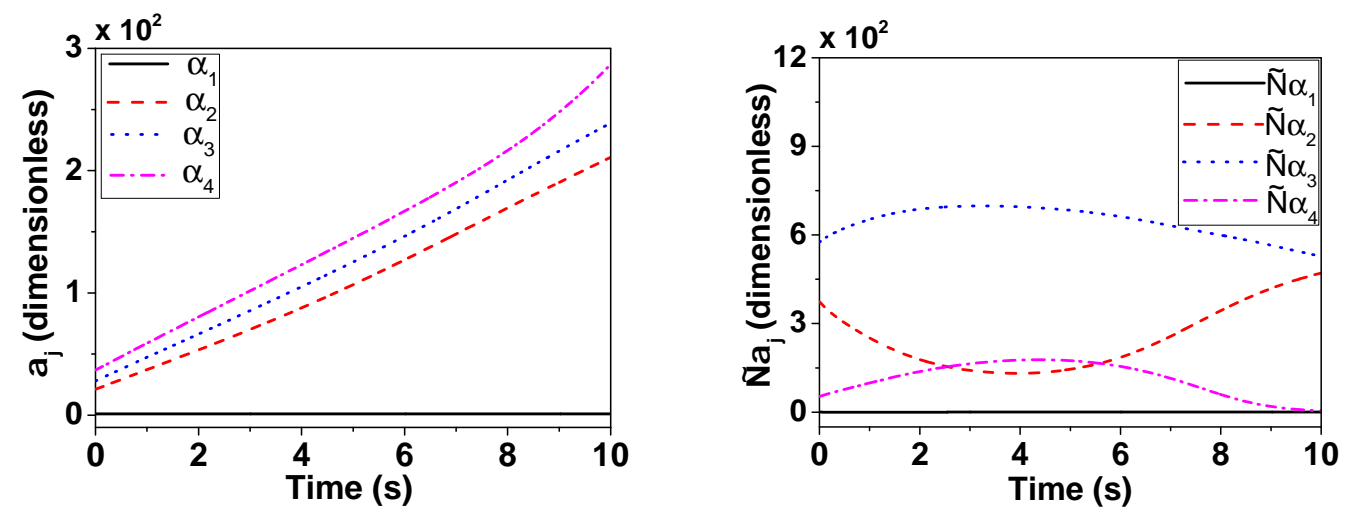

Figure 8: Evolution of the particle masses $\alpha_{j}$ (left panel) and the corresponding number of particles $\widetilde{N}_{\alpha_{j}}$ (right panel) using MPM under pure growth. The PSD at $t=0 \mathrm{~s}$ in Fig. 7 (continuous line) is supplied as the initial condition. A total of four particle masses are used to approximate the PSD.
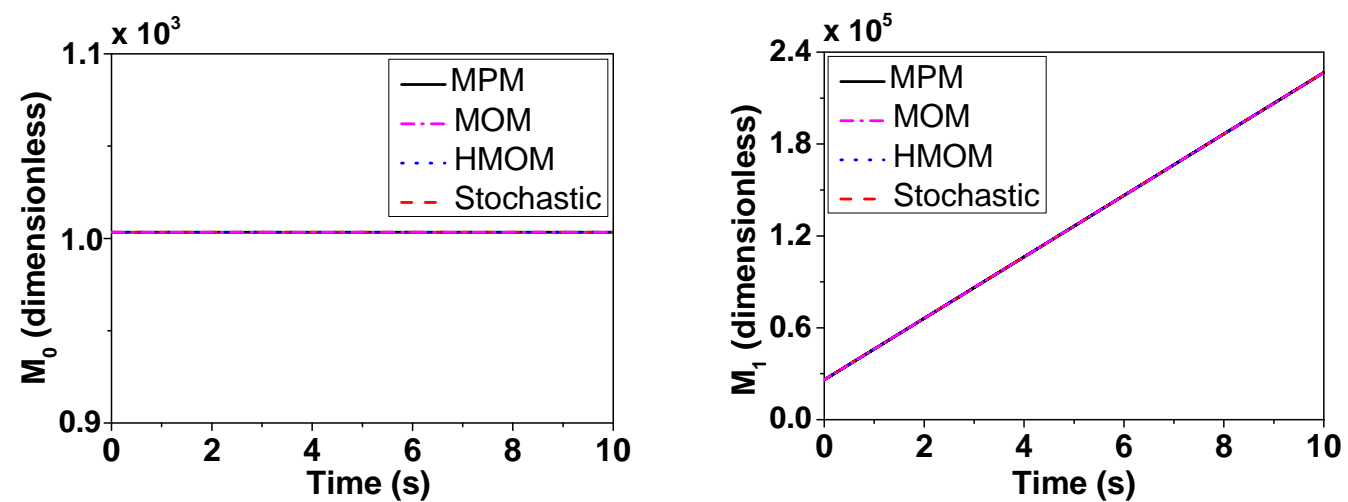

Figure 9: Comparison of the zeroth moment $M_{0}$ (left panel) and the first moment $M_{1}$ (right panel) between MPM, MOM, HMOM and the stochastic method under pure growth.

particle mass, $\alpha_{1}$, to be equal to the smallest mass so that the corresponding number of particles, $\widetilde{N}_{\alpha_{1}}$, can be used as an approximation of $N_{1}$ of the true PSD. 
Case 5 A uniform distribution:

$$
N(i, t=0)=10, \quad i=1,2, \ldots, 30 .
$$

Case 3 A unimodal distribution:

$$
N(i=30, t=0)=100 .
$$

Case 4 A parabolic distribution:

$$
N(i, t=0)=300 i-10 i^{2}, \quad i=1,2, \ldots, 30 .
$$


To determine the error in the moments computed using MPM the following relative error metric is used:

$$
M_{k, \text { error }}=\frac{\left|\widetilde{M}_{k}-M_{k}\right|}{M_{k}+\eta},
$$

where $\widetilde{M}_{k}$ is the $k$-th order moment calculated using MPM while $M_{k}$ is from a high-precision stochastic solution. $\eta$ is a constant assumed to be 1 . The purpose of introducing $\eta$ is to prevent the error metric from tending towards infinity because as particles shrink and are removed from the system $M_{k}$ would tend towards zero.

For Case 1, a log-normal distribution is supplied as the initial condition. Evolution of the PSD computed by solving the master equation under pure shrinkage is shown in Fig. 10. The distribution shifts towards the smallest particle mass and at $t=2 \mathrm{~s}$ all the particles have been removed from the system.

The simulation results using MPM where five particle masses $\left(N_{\mathrm{p}}=5\right)$ are used to approximate the PSD are shown in Fig. 11. $\alpha_{j}(j=2,3,4,5)$ move towards the smallest particle mass before flattening out as almost all the particles have been removed. Large particles shrink to form smaller ones, therefore, $\widetilde{N}_{\alpha_{j}}(j=2,3,4,5)$ decreases while $\widetilde{N}_{\alpha_{1}}$ increases. However, once the rate of removal of the smallest particles is greater than the rate of formation from large particles $\widetilde{N}_{\alpha_{1}}$ also decreases.

The relative error for moments of order $k=0$ to $8\left(N_{\mathrm{p}}=5 ; k=0, \ldots, 2 N_{\mathrm{p}}-2\right)$ using MPM is shown in Fig. 12. The errors gradually increase over time as the moments tend towards zero. However, at $t=1 \mathrm{~s}$, when almost no particles are left in the system, the errors are at most $\sim 10 \%$. 


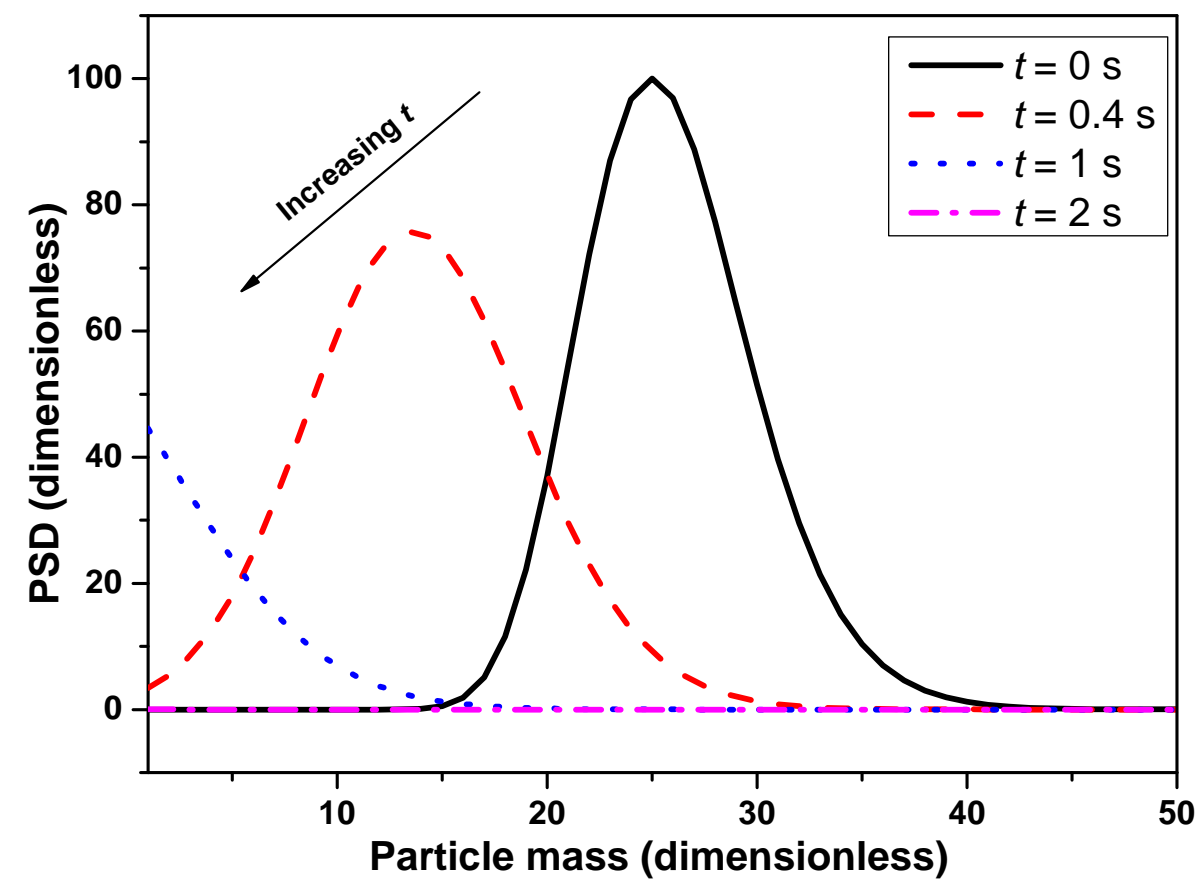

Figure 10: Evolution of the PSD computed by solving the master equation under pure shrinkage (Case 1).

To investigate the influence of the number of particle masses, $N_{\mathrm{p}}$, on the accuracy of MPM, $N_{\mathrm{p}}$ is varied from 3 to 5 . (We see little decrease in the error for $N_{\mathrm{p}}>5$.) The zeroth and first moments computed using MPM for different $N_{\mathrm{p}}$ are compared with the stochastic solution in Fig. 13. $\widetilde{M}_{0}$ computed using MPM for $N_{\mathrm{p}}=3$ (dashed line) shows an obvious discrepancy with $M_{0}$ computed using the stochastic method (continuous line). By contrast, the results obtained using $N_{\mathrm{p}}=4$ and 5 show a good agreement with the stochastic solution. $\widetilde{M}_{1}$ does not display any sensitivity to $N_{\mathrm{p}}$. The timeaveraged ( $t=0$ to $1.5 \mathrm{~s}$ ) relative moment error, $M_{k \text {,error }}$, is shown in Table 2 as a function of $N_{\mathrm{p}}$ and $k$. A higher accuracy is observed when larger values of $N_{\mathrm{p}}$ are used; the errors show about an order of magnitude decrease when $N_{\mathrm{p}}$ is 

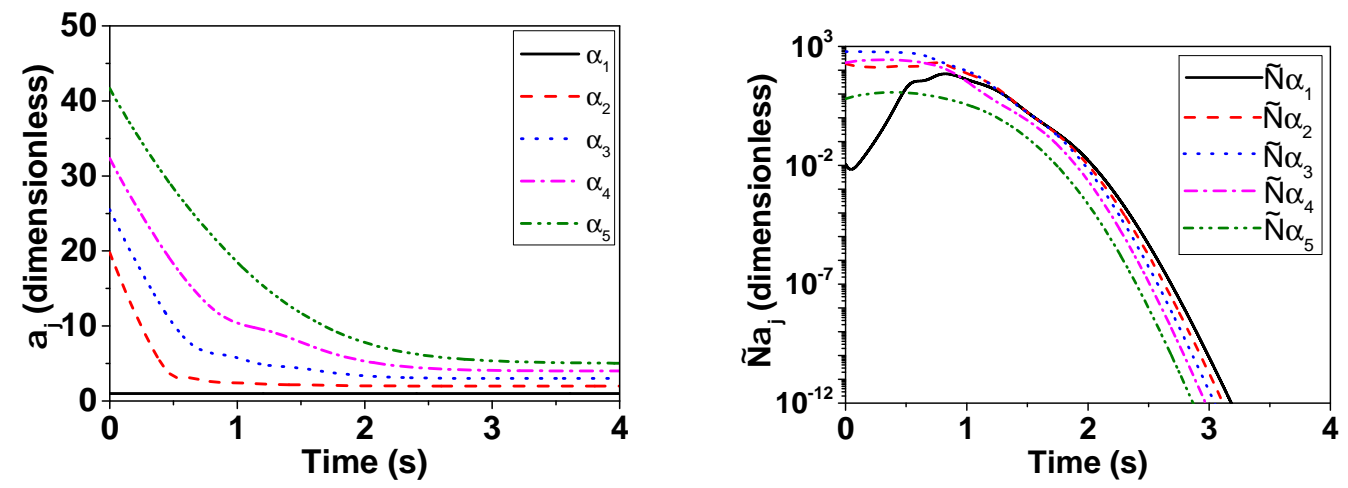

Figure 11: Evolution of the particle masses $\alpha_{j}$ (left panel) and the corresponding number of particles $\widetilde{N}_{\alpha_{j}}$ (right panel) using MPM under pure shrinkage. The PSD at $t=0 \mathrm{~s}$ in Fig. 10 (continuous line) is supplied as the initial condition (Case 1). A total of five particle masses are used to approximate the PSD.

increased from 3 to 5 . As more particle masses are used, the approximation made on the pointwise value of the $\operatorname{PSD}\left(\widetilde{N}_{\alpha_{1}} \approx N_{1}\right)$ is closer to the real value. However, the higher-order moments tend to exhibit a larger error than lower-order moments. As can be seen in Fig. 12, errors in the higherorder moments are initially small; however, as the simulation proceeds, the moments tend towards zero making the relative errors large. Nevertheless, these errors decrease significantly with an increase $N_{\mathrm{p}}$. For example, $M_{4, \text { error }}$ decreases from 0.3088 to 0.2053 when $N_{\mathrm{p}}$ is increased from 3 to 4 , and $M_{6 \text {,error }}$ decreases from 0.3515 to 0.2522 when $N_{\mathrm{p}}$ is increased from 4 to 5 .

The ability of different methods to handle shrinkage can be seen in Fig. 14. MOM does not account for the consumption of particles due to shrinkage therefore $\widetilde{M}_{0}$ remains constant; however, the behaviour of $\widetilde{M}_{1}$ is somewhat more reasonable. $\widetilde{M}_{1}$ is set to be equal to $\widetilde{M}_{0}$ whenever $\widetilde{M}_{1}$ falls below $\widetilde{M}_{0}$ to ensure that the moments are strictly monotonic. HMOM performs 


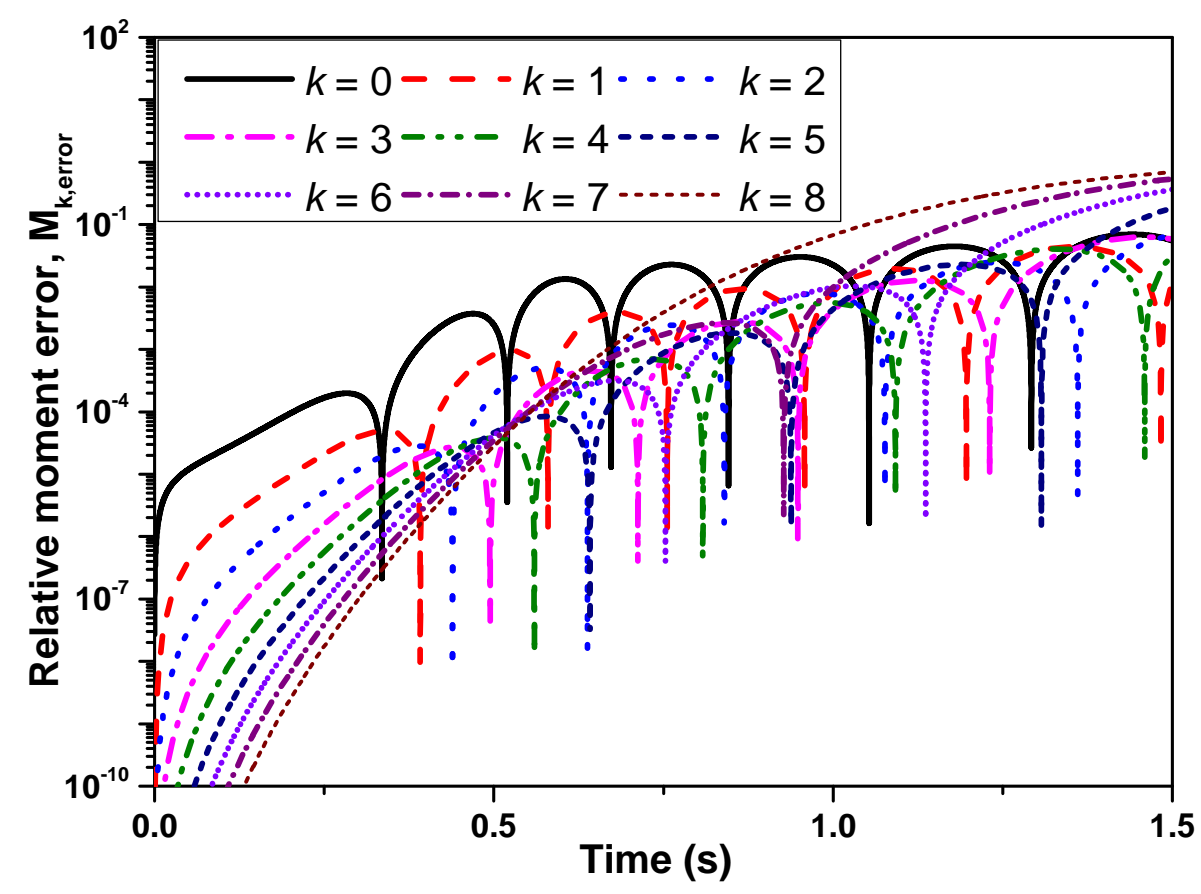

Figure 12: Error in the $k$-th order moment using MPM relative to a high-precision stochastic solution under pure shrinkage. Errors correspond to Case 1 where a log-normal distribution is supplied as the initial condition.

much better as it includes a source term to account for the consumption of the smallest particles. As large particles shrink to eventually form the smallest particles, it was assumed that the number of the smallest particles formed from the large particles is proportional to the mass lost from the large particles [39] (see Appendix B). This assumption is too coarse. Initially, the mass of large particles can decrease without there being a change in the number of particles. HMOM overestimates the number of the smallest particles, and therefore $M_{0}$. However, small particles are easier to remove; therefore, the trend reverses and HMOM underestimates $M_{0}$ (and $M_{1}$ ). By contrast, the moments computed using MPM for $N_{\mathrm{p}}=4$ shows an excellent 

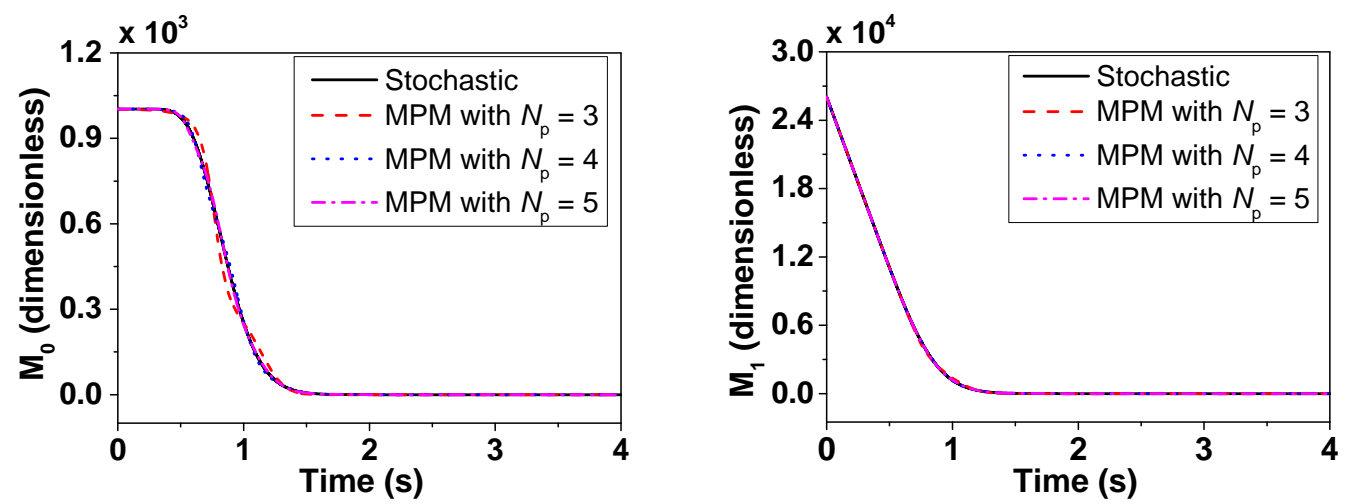

Figure 13: Sensitivity of the zeroth moment $M_{0}$ (left panel) and the first moment $M_{1}$ (right panel) to the number of particle masses, $N_{p}$, using MPM under pure shrinkage. Results correspond to Case 1 where a log-normal distribution is supplied as the initial condition. The stochastic solution is shown as a point of reference.

agreement with the stochastic solution.
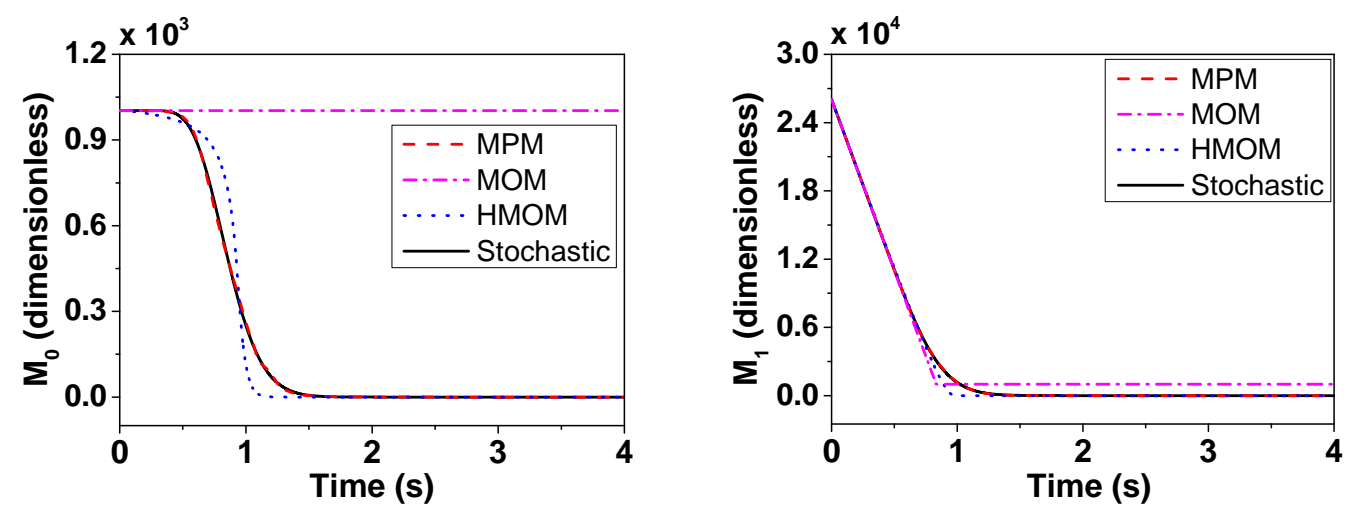

Figure 14: Comparison of the zeroth moment $M_{0}$ (left panel) and the first moment $M_{1}$ (right panel) between MPM (four particle masses), MOM, HMOM and the stochastic method under pure shrinkage. Results correspond to Case 1 where a log-normal distribution is supplied as the initial condition. 
Table 2: Average error in the $k$-th order moment using MPM relative to a high-precision stochastic solution, for different number of particle masses, $N_{p}$, under pure shrinkage. Errors correspond to Case 1 where a log-normal distribution is supplied as the initial condition.

\begin{tabular}{lcll}
\hline $\mathrm{k}$ & $N_{\mathrm{p}}=3$ & $N_{\mathrm{p}}=4$ & $N_{\mathrm{p}}=5$ \\
\hline 0 & 0.0912 & 0.0304 & 0.0104 \\
1 & 0.1179 & 0.0399 & 0.0103 \\
2 & 0.1711 & 0.0793 & 0.0201 \\
3 & 0.2362 & 0.1393 & 0.0548 \\
4 & 0.3088 & 0.2053 & 0.1123 \\
5 & - & 0.2767 & 0.1802 \\
6 & - & 0.3515 & 0.2522 \\
7 & - & - & 0.3269 \\
8 & - & - & 0.4041 \\
\hline
\end{tabular}

For Case 2, another lognormal distribution is adopted where the average particle mass is about three orders-of-magnitude larger than the smallest particle mass. Figure 15 compares the zeroth and first order moments computed using MPM for different $N_{\mathrm{p}}$ and the stochastic method. Compared with Case 1, MPM performs relatively poorly. $\widetilde{M}_{0}$ obtained using MPM for $N_{\mathrm{p}}=3$ and 4 do not match the stochastic solution well. However the discrepancy becomes less obvious with each increase in $N_{\mathrm{p}}$ suggesting that estimation of the boundary flux term is closer to the real solution. By contrast, $\widetilde{M}_{1}$ obtained using MPM shows an excellent agreement with the stochastic solution. 

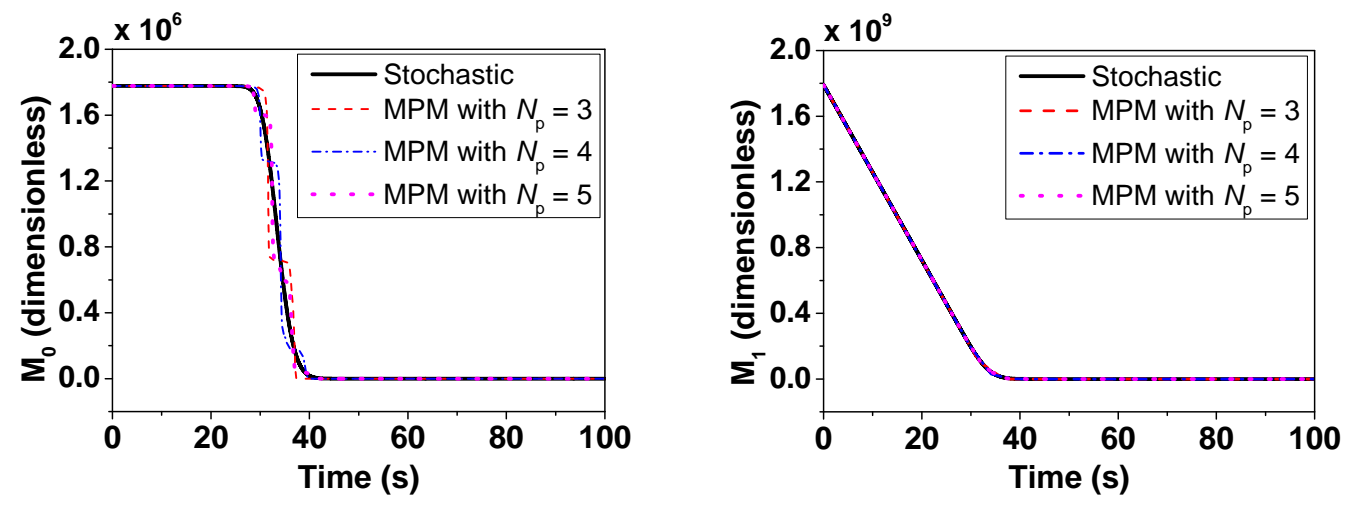

Figure 15: Sensitivity of the zeroth moment $M_{0}$ (left panel) and the first moment $M_{1}$ (right panel) to the number of particle masses, $N_{p}$, using MPM under pure shrinkage. Results correspond to Case 2, where a log-normal distribution is supplied as the initial condition. The average particle mass is about three orders-of-magnitude larger than the smallest particle mass. The stochastic solution is shown as a point of reference.

Table 3 lists the time-averaged relative moment errors for Case 2. In general, the moment errors are larger than for Case 1. This is because the PSD spans a much larger mass range than in Case 1, which makes it numerically more challenging for MPM to approximate the boundary flux term accurately. However, the moment errors show a systematic decrease with each increase in $N_{\mathrm{p}}$.

Figure 16 compares the zeroth and first order moments obtained by different methods for Case 2. Again, MOM could not predict the decrease in the number of particles, and HMOM exhibits very large moment errors due to the overestimation of the formation of the smallest particles. Although MPM does not show as high accuracy as it does for Case 1, it is still the most accurate among the moment methods. 
Table 3: Average error in the $k$-th order moment using MPM relative to a high-precision stochastic solution, for different number of particle masses, $N_{p}$, under pure shrinkage. Errors correspond to Case 2 where a lognormal distribution is supplied as the initial condition. The average particle mass is about three ordersof-magnitude larger than the smallest particle mass.

\begin{tabular}{lcll}
\hline $\mathrm{k}$ & $N_{\mathrm{p}}=3$ & $N_{\mathrm{p}}=4$ & $N_{\mathrm{p}}=5$ \\
\hline 0 & 0.1406 & 0.1262 & 0.0918 \\
1 & 0.1472 & 0.1285 & 0.0921 \\
2 & 0.2020 & 0.1488 & 0.1099 \\
3 & 0.2842 & 0.1758 & 0.1544 \\
4 & 0.3408 & 0.2364 & 0.1823 \\
5 & - & 0.3390 & 0.2122 \\
6 & - & 0.3733 & 0.2934 \\
7 & - & - & 0.3757 \\
8 & - & - & 0.4387 \\
\hline
\end{tabular}

The results for Case 3 where a unimodal distribution is supplied as the initial condition are similar to Case 1 and are shown in Fig. 17 and Table 4. For Case 4, a parabolic distribution is supplied as the initial condition. Figure 18 shows that $\widetilde{M}_{0}$ computed using MPM for $N_{\mathrm{p}}=3$ shows a poor agreement with the stochastic solution. Even if $N_{\mathrm{p}}$ is increased to 4, a slight discrepancy can still be observed. A satisfactory agreement is obtained when $N_{\mathrm{p}}$ is increased to 5 . The conclusions drawn from the corresponding average relative error in Table 5 are similar to those for previous cases. For Case 5 , a uniform distribution is supplied as the initial condition. The results are 

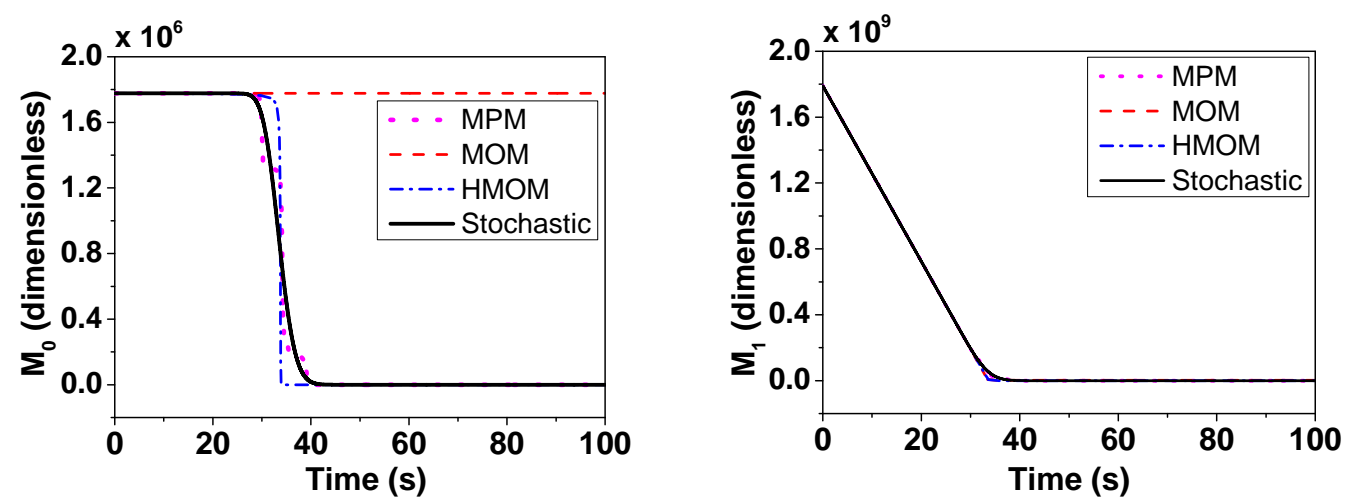

Figure 16: Comparison of the zeroth moment $M_{0}$ (left panel) and the first moment $M_{1}$ (right panel) between MPM (four particle masses), MOM, HMOM and the stochastic method under pure shrinkage. Results correspond to Case 2 where a log-normal distribution is supplied as the initial condition. The average particle mass is about three orders-of-magnitude larger than the smallest particle mass.

similar to those for Case 4 and are shown in Fig. 19 and Table 6.

Based on the five cases considered above, we conclude that MPM is able to simulate the shrinkage of different types of PSDs as long as a sufficient number of particle masses are used. $N_{p}=4$ is a good compromise between accuracy and computational efficiency.

\subsection{Combined processes}

We looked at the processes of inception, coagulation, growth and shrinkage in isolation. Now we test MPM against MOM, HMOM and the stochastic method for all of these processes combined. Two types of PSDs are supplied as the initial condition and the shrinkage kernel is varied to simulate relatively weak (Case 7) and strong (Case 8) shrinkage: 

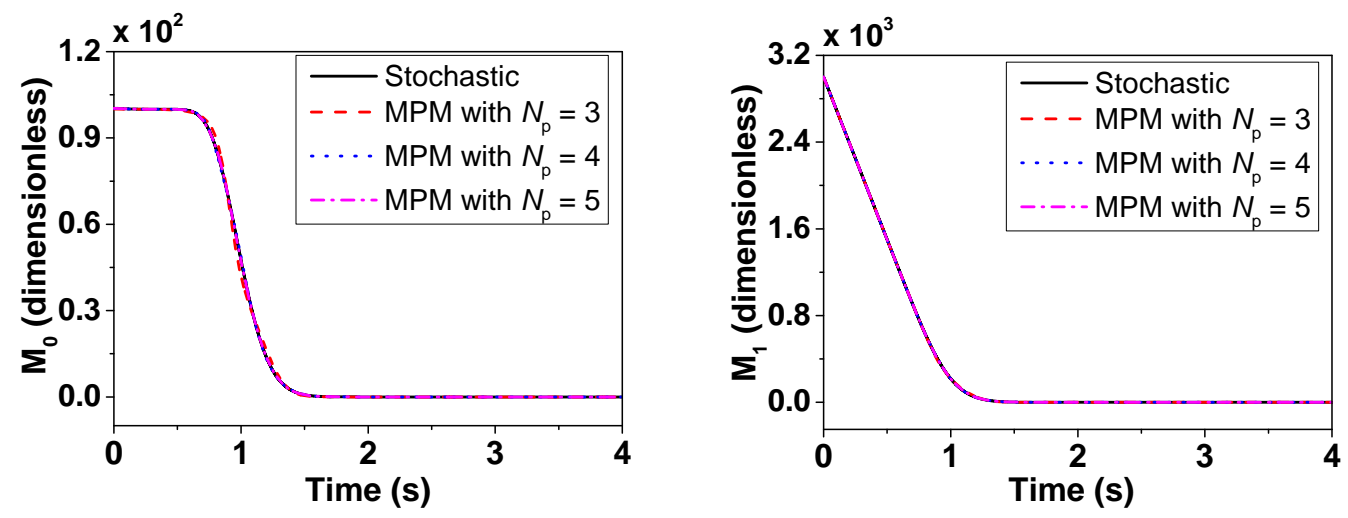

Figure 17: Sensitivity of the zeroth moment $M_{0}$ (left panel) and the first moment $M_{1}$ (right panel) to the number of particle masses, $N_{p}$, using MPM under pure shrinkage. Results correspond to Case 3 where a unimodal distribution is supplied as the initial condition. The stochastic solution is shown as a point of reference.
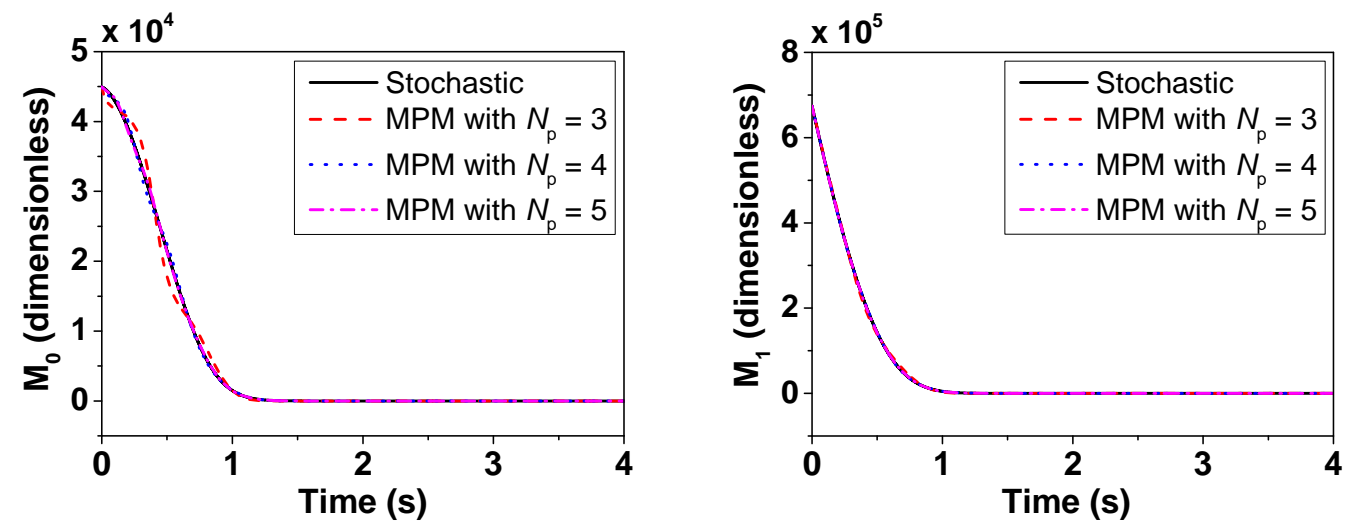

Figure 18: Sensitivity of the zeroth moment $M_{0}$ (left panel) and the first moment $M_{1}$ (right panel) to the number of particle masses, $N_{p}$, using MPM under pure shrinkage. Results correspond to Case 4 where a parabolic distribution is supplied as the initial condition. The stochastic solution is shown as a point of reference. 
Table 4: Average error in the $k$-th order moment using MPM relative to a high-precision stochastic solution, for different number of particle masses, $N_{p}$, under pure shrinkage. Errors correspond to Case 3 where a unimodal distribution is supplied as the initial condition.

\begin{tabular}{cccc}
\hline $\mathrm{k}$ & $N_{\mathrm{p}}=3$ & $N_{\mathrm{p}}=4$ & $N_{\mathrm{p}}=5$ \\
\hline 0 & 0.0256 & 0.0053 & 0.0009 \\
1 & 0.0366 & 0.0057 & 0.0008 \\
2 & 0.0701 & 0.0143 & 0.0014 \\
3 & 0.1158 & 0.0381 & 0.0049 \\
4 & 0.1667 & 0.0756 & 0.0170 \\
5 & - & 0.1206 & 0.0408 \\
6 & - & 0.1689 & 0.0749 \\
7 & - & - & 0.1163 \\
8 & - & - & 0.1615 \\
\hline
\end{tabular}

Case 6 Inception kernel $I_{m_{1}}=100 \mathrm{~s}^{-1}$, growth kernel $K_{\mathrm{G}}=20 \mathrm{~s}^{-1}$, coagulation kernel $K_{\mathrm{Cg}}=2 \times 10^{-4} \mathrm{~s}^{-1}$ and shrinkage kernel $K_{\mathrm{Sk}}=30 \mathrm{~s}^{-1}$ with a log-normal distribution as the initial condition (see Eq. (12)):

$$
N(i, t=0)=100 \exp \left(-(\log (i)-\log (25))^{2} / 0.05\right), \quad i=1,2, \ldots, 100 .
$$

Case $7 I_{m_{1}}=100 \mathrm{~s}^{-1}, K_{\mathrm{G}}=20 \mathrm{~s}^{-1}, K_{\mathrm{Cg}}=2 \times 10^{-4} \mathrm{~s}^{-1}$ and $K_{\mathrm{Sk}}=22 \mathrm{~s}^{-1}$ with a unimodal distribution as the initial condition (see Eq. (20)):

$$
N(i=30, t=0)=100 .
$$

Case $8 I_{m_{1}}=100 \mathrm{~s}^{-1}, K_{\mathrm{G}}=20 \mathrm{~s}^{-1}, K_{\mathrm{Cg}}=2 \times 10^{-4} \mathrm{~s}^{-1}$ and $K_{\mathrm{Sk}}=30 \mathrm{~s}^{-1}$ 
Table 5: Average error in the $k$-th order moment using MPM relative to a high-precision stochastic solution, for different number of particle masses, $N_{p}$, under pure shrinkage. Errors correspond to Case 4 where a parabolic distribution is supplied as the initial condition.

\begin{tabular}{ccll}
\hline $\mathrm{k}$ & $N_{\mathrm{p}}=3$ & $N_{\mathrm{p}}=4$ & $N_{\mathrm{p}}=5$ \\
\hline 0 & 0.1456 & 0.0512 & 0.0088 \\
1 & 0.1605 & 0.0665 & 0.0126 \\
2 & 0.1965 & 0.0981 & 0.0261 \\
3 & 0.2413 & 0.1383 & 0.0501 \\
4 & 0.2912 & 0.1827 & 0.0832 \\
5 & - & 0.2294 & 0.1226 \\
6 & - & 0.2775 & 0.1659 \\
7 & - & - & 0.2113 \\
8 & - & - & 0.2577 \\
\hline
\end{tabular}

with a unimodal distribution as the initial condition (see Eq. (20)):

$$
N(i=30, t=0)=100 .
$$

For Case 6, the shrinkage kernel is larger than the growth kernel, therefore, there is a net shrinkage of particles and the PSD shifts towards the smallest particle mass as shown in Fig. 20. By the end of simulation $(t=10 \mathrm{~s})$, no particles are left in the system. MOM predicts a slight decrease in $\widetilde{M}_{0}$ as shown in Fig. 21 due to the interplay between inception and coagulation. $\widetilde{M}_{1}$ computed using MOM decreases much faster than the stochastic solution. As we saw in the Section 3.4, $\widetilde{M}_{1}$ would eventually fall below $\widetilde{M}_{0}$ under 

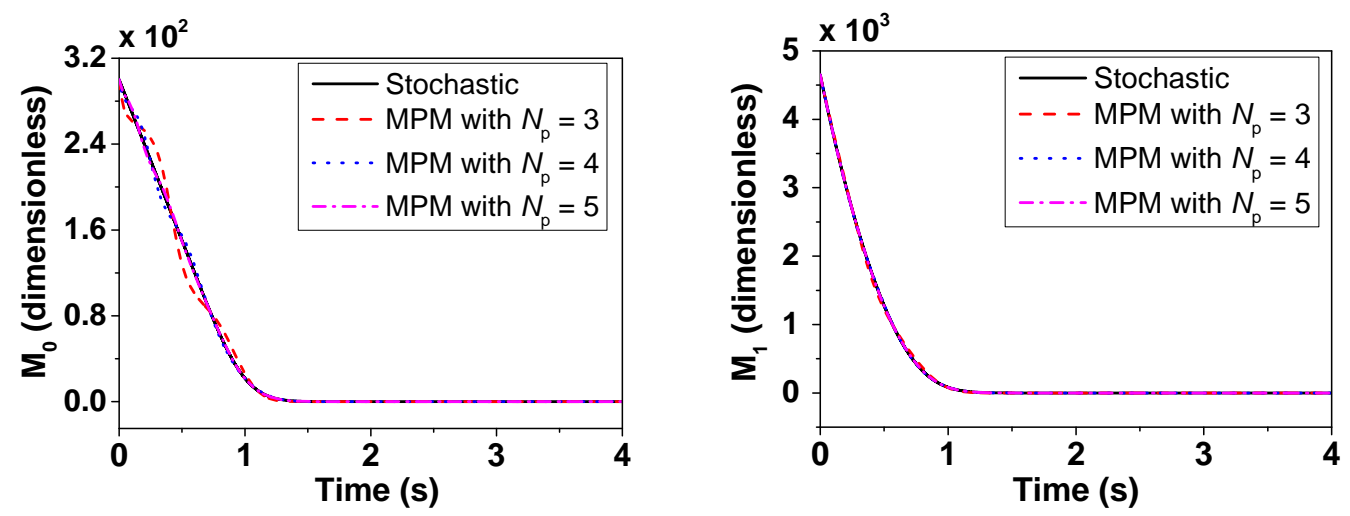

Figure 19: Sensitivity of the zeroth moment $M_{0}$ (left panel) and the first moment $M_{1}$ (right panel) to the number of particle masses, $N_{p}$, using MPM under pure shrinkage. Results correspond to Case 5 where a uniform distribution is supplied as the initial condition. The stochastic solution is shown as a point of reference.

pure shrinkage as the MOM formulation does not include a source term to account for the consumption of particles due to shrinkage. To maintain the monotonicity of moments, from about $t=2.5 \mathrm{~s}$ onwards, $\widetilde{M}_{1}$ is set to be equal to $\widetilde{M}_{0}$. HMOM reproduces the decreasing trend in $M_{0}$ and $M_{1}$, however, there is an obvious discrepancy compared with the stochastic solution. By contrast, $\widetilde{M}_{0}$ and $\widetilde{M}_{1}$ obtained using MPM for $N_{\mathrm{p}}=4$ is in a much better agreement with the stochastic solution compared with MOM and HMOM.

For Case 7, a unimodal distribution where its mode is located at a mass of 30 evolves into a bimodal distribution under the combined effects of inception, coagulation, growth and shrinkage as shown in Fig. 22. There is only a slight shift in the position of the second mode of the distribution because the shrinkage kernel is only slightly larger than the growth kernel. As shown in Fig. 23, $\widetilde{M}_{0}$ and $\widetilde{M}_{1}$ computed using MPM show a good agreement with the 
Table 6: Average error in the $k$-th order moment using MPM relative to a high-precision stochastic solution, for different number of particle masses, $N_{p}$, under pure shrinkage. Errors correspond to Case 5 where a uniform distribution is supplied as the initial condition

\begin{tabular}{lcll}
\hline $\mathrm{k}$ & $N_{\mathrm{p}}=3$ & $N_{\mathrm{p}}=4$ & $N_{\mathrm{p}}=5$ \\
\hline 0 & 0.0642 & 0.0156 & 0.0036 \\
1 & 0.0795 & 0.0168 & 0.0023 \\
2 & 0.1218 & 0.0369 & 0.0046 \\
3 & 0.1735 & 0.0734 & 0.0148 \\
4 & 0.2294 & 0.1192 & 0.0368 \\
5 & - & 0.1689 & 0.0699 \\
6 & - & 0.2203 & 0.1109 \\
7 & - & - & 0.1565 \\
8 & - & - & 0.2043 \\
\hline
\end{tabular}

stochastic solution while MOM and HMOM fail to even match. The performance of MOM and HMOM is similar to Case 6 except that MOM predicts a nonlinear increase in $\widetilde{M}_{0}$. This shows that while inception is dominant, nonlinear effects from coagulation is significant.

For Case 8 , the shrinkage kernel, $K_{\mathrm{Sk}}$, is increased to $30 \mathrm{~s}^{-1}$ while the inception, coagulation and growth kernels are the same as in Case 7 . A bimodal distribution is again observed in Fig. 24. This time however the PSD shifts towards smaller particle masses at a much faster speed within the same period of time, simulating a situation with a strong particle shrinkage. Comparison of $M_{0}$ and $M_{1}$ between the different methods is shown in Fig. 25 


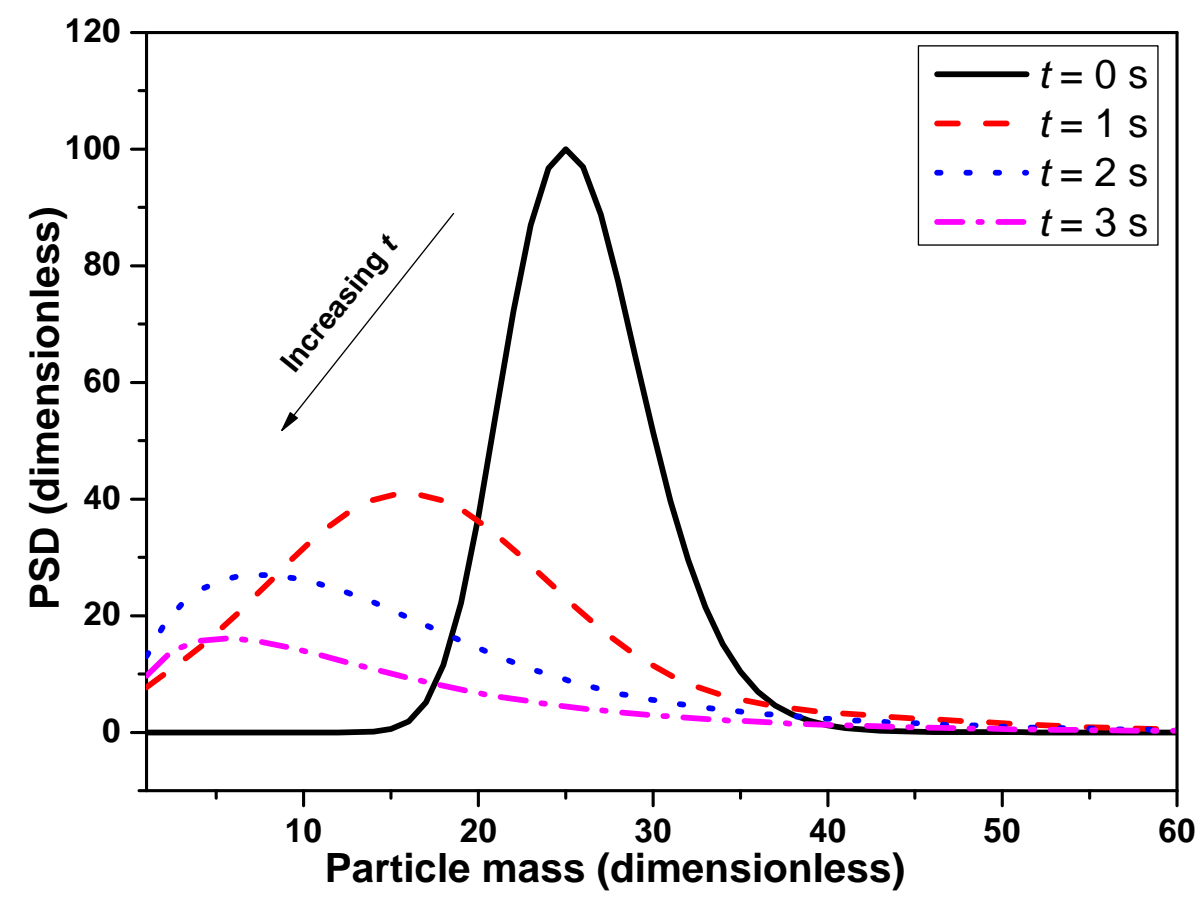

Figure 20: Evolution of the PSD computed using the stochastic method under all particle processes (Case 6).

446 and the conclusion that can be drawn is similar to Case 7 . 

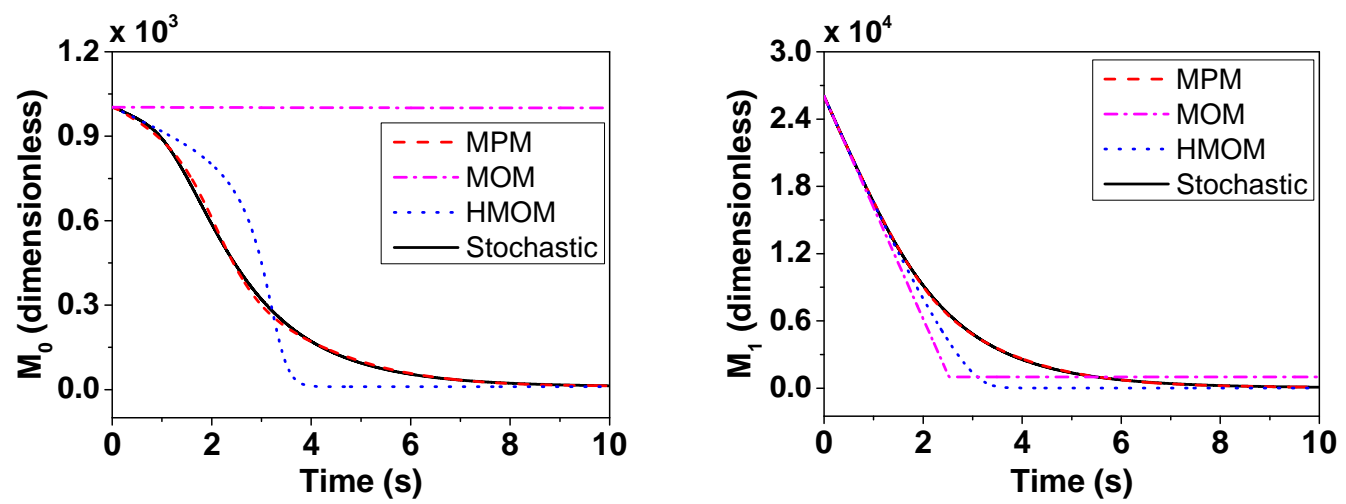

Figure 21: Comparison of the zeroth moment $M_{0}$ (left panel) and the first moment $M_{1}$ (right panel) between MPM, MOM, HMOM and the stochastic method under all particle processes. Results correspond to Case 6 where a log-normal distribution is supplied as the initial condition.

\section{Conclusion}

A new moment projection method (MPM) for solving the population balance equation (PBE) has been developed and presented. The main advantages of this method are its ease of implementation and numerical robustness as well as its ability to deal with particle shrinkage. It directly solves the moment transport equation for the moments so that the source terms can be readily evaluated using the method of moments with interpolative closure (MOMIC). A set of particle masses are used to approximate the discretemass distribution where one of the particle masses is fixed at the smallest particle. The algorithm by Blumstein and Wheeler is used to track the number of these particles which eliminates the need for matrix inversion which can lead to singularity problems. The new method is compared with the method of moments (MOM) and the hybrid method of moments (HMOM), 


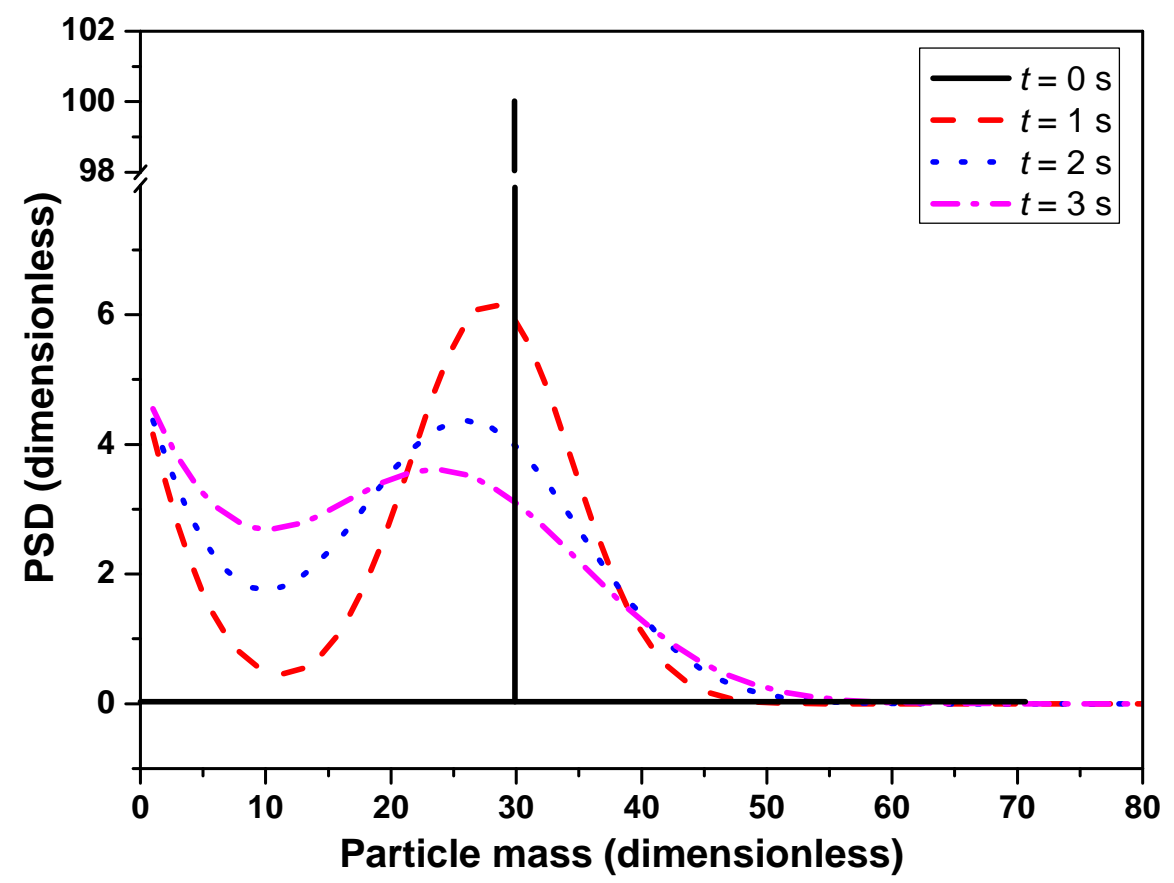

Figure 22: Evolution of the PSD computed using the stochastic method under all particle processes but with relatively weak shrinkage (Case 7).

first for the individual processes of particle inception, coagulation, growth and shrinkage (constant kernels), then for all of these processes combined; different types of particles size distributions (PSDs) are supplied as an initial condition. It is shown that MPM is just as accurate as MOM and HMOM when used to treat inception, coagulation and growth. However, when it comes to shrinkage, MPM performs much better than MOM and HMOM. The accuracy of MPM improves with the number of particle masses, $N_{\mathrm{p}}$, and $N_{\mathrm{p}}=5$ is found to provide an excellent agreement with a high-precision stochastic solution calculated using the direct simulation algorithm (DSA). Higher-order moments computed using MPM show larger relative errors than 

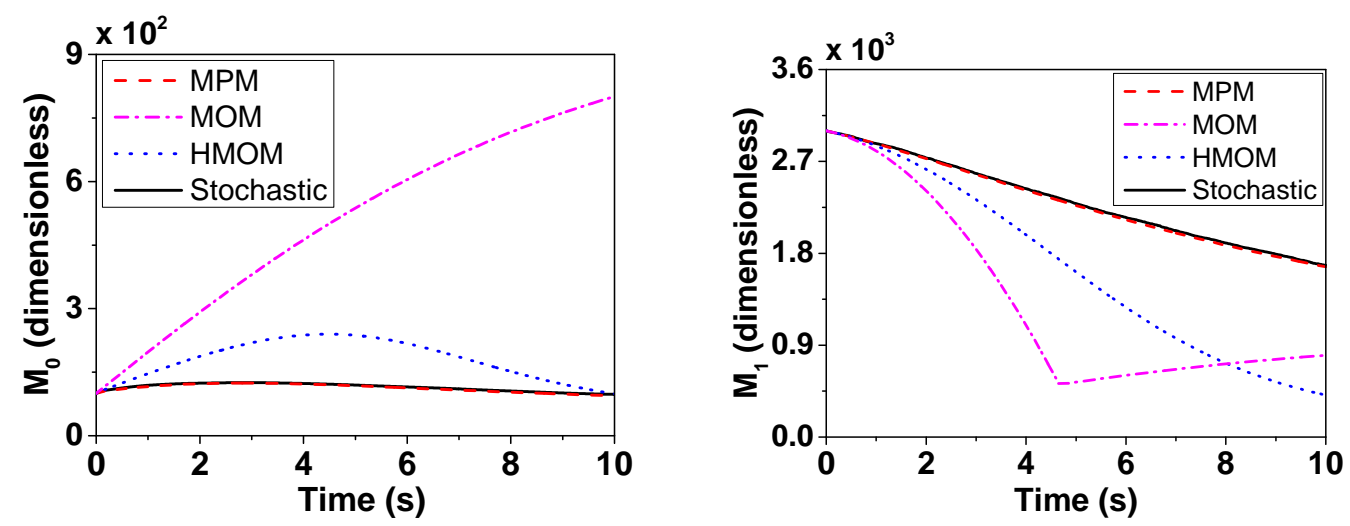

Figure 23: Comparison of the zeroth moment $M_{0}$ (left panel) and the first moment $M_{1}$ (right panel) between MPM, MOM, HMOM and the stochastic method under all particle processes. Results correspond to Case 7 where a unimodal distribution is supplied as the initial condition and shrinkage is relatively weak.

lower-order moments consistent with other moment methods. These errors gradually increase with time because the moments tend towards zero. As fragmentation (or breakage) is a quite a common phenomena, future work includes extension of MPM to include the fragmentation process. The performance of the method using physically realistic Brownian kernels is also to be investigated.

\section{Acknowledgement}

This research is supported by the National Research Foundation, Prime Minister's Office, Singapore under its CREATE programme. 


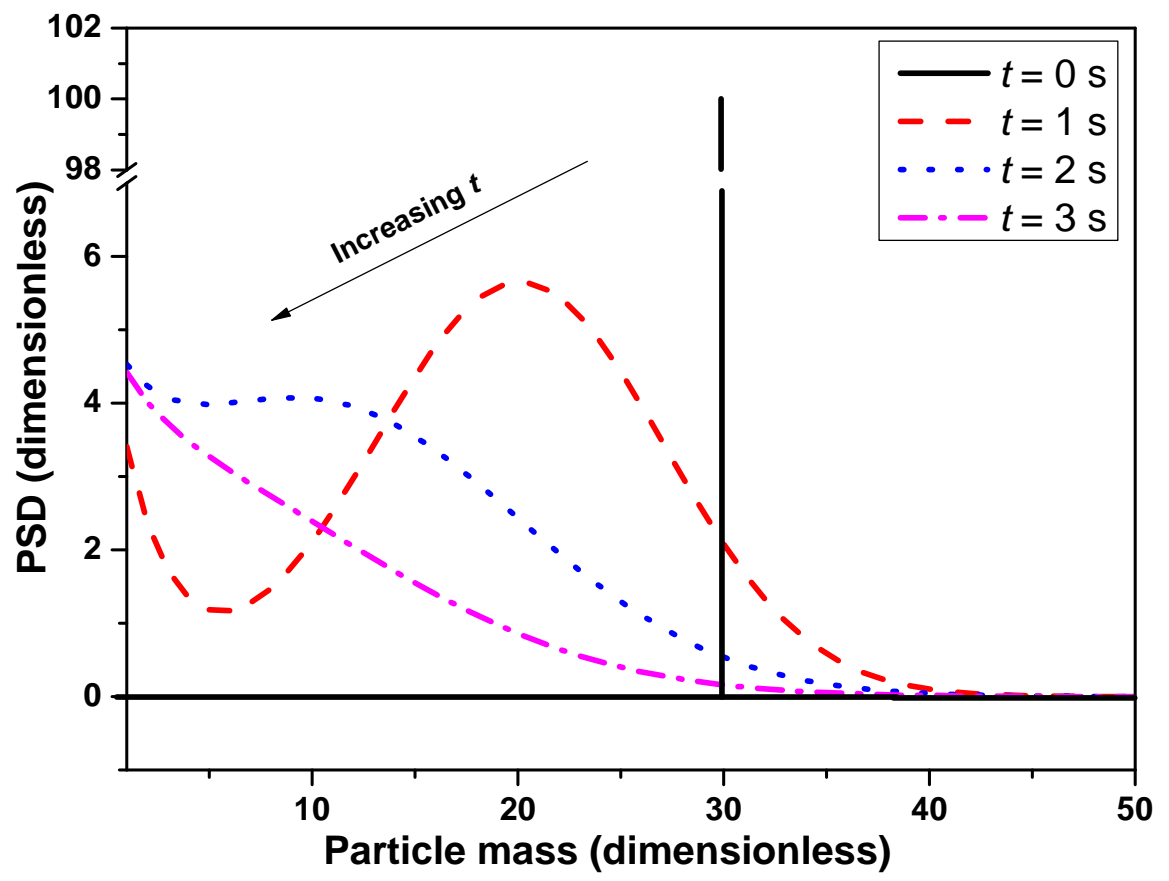

Figure 24: Evolution of the PSD computed using the stochastic method under all particle processes but with relatively strong shrinkage (Case 8).

\section{Nomenclature}

Upper-case Roman

$G$ Source term due to coagulation

${ }_{480} \quad I$ Inception rate

$K_{\mathrm{Cg}} \quad$ Coagulation kernel

$K_{\mathrm{G}} \quad$ Growth kernel

$K_{\text {Sk }} \quad$ Shrinkage kernel 

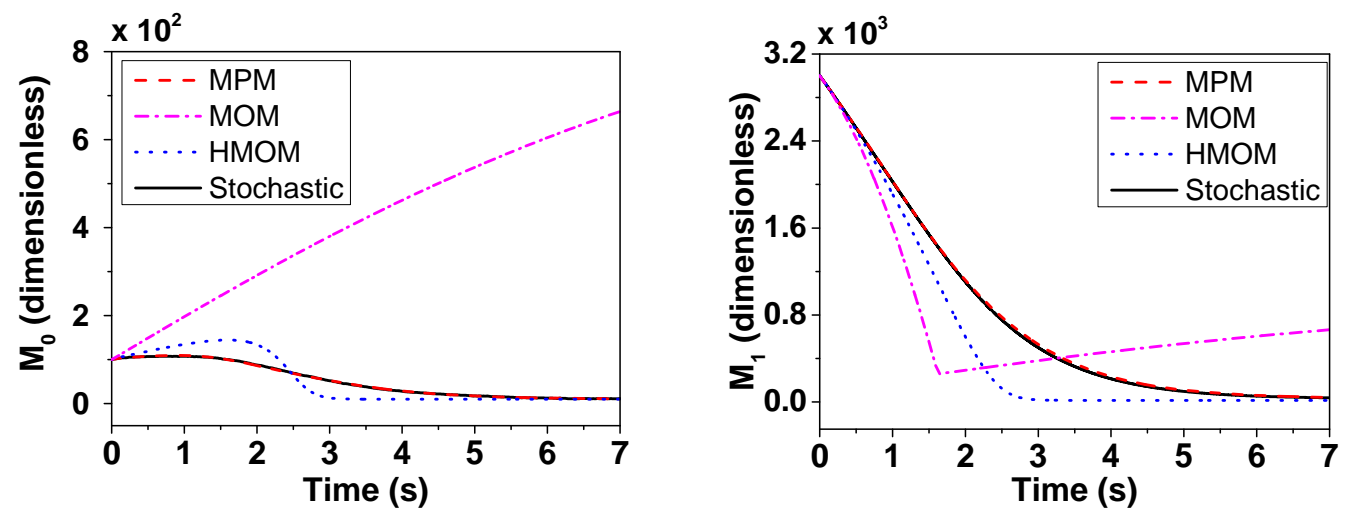

Figure 25: Comparison of the zeroth moment $M_{0}$ (left panel) and the first moment $M_{1}$ (right panel) between MPM, MOM, HMOM and the stochastic method under all particle processes. Results correspond to Case 8 where a unimodal distribution is supplied as the initial condition and shrinkage is relatively strong.

M Moment

$N \quad$ Number

$\mathbf{P}$ Symmetric tridiagonal matrix which is a function of recursion coefficients $a$ and $b$

$R$ Source term due to inception

481

$S$ Source term due to shrinkage

$W \quad$ Source term due to growth

Z Matrix with components $Z$ which are a function of the moments $M$

Lower-case Roman

$a, b \quad$ Recursion coefficients 


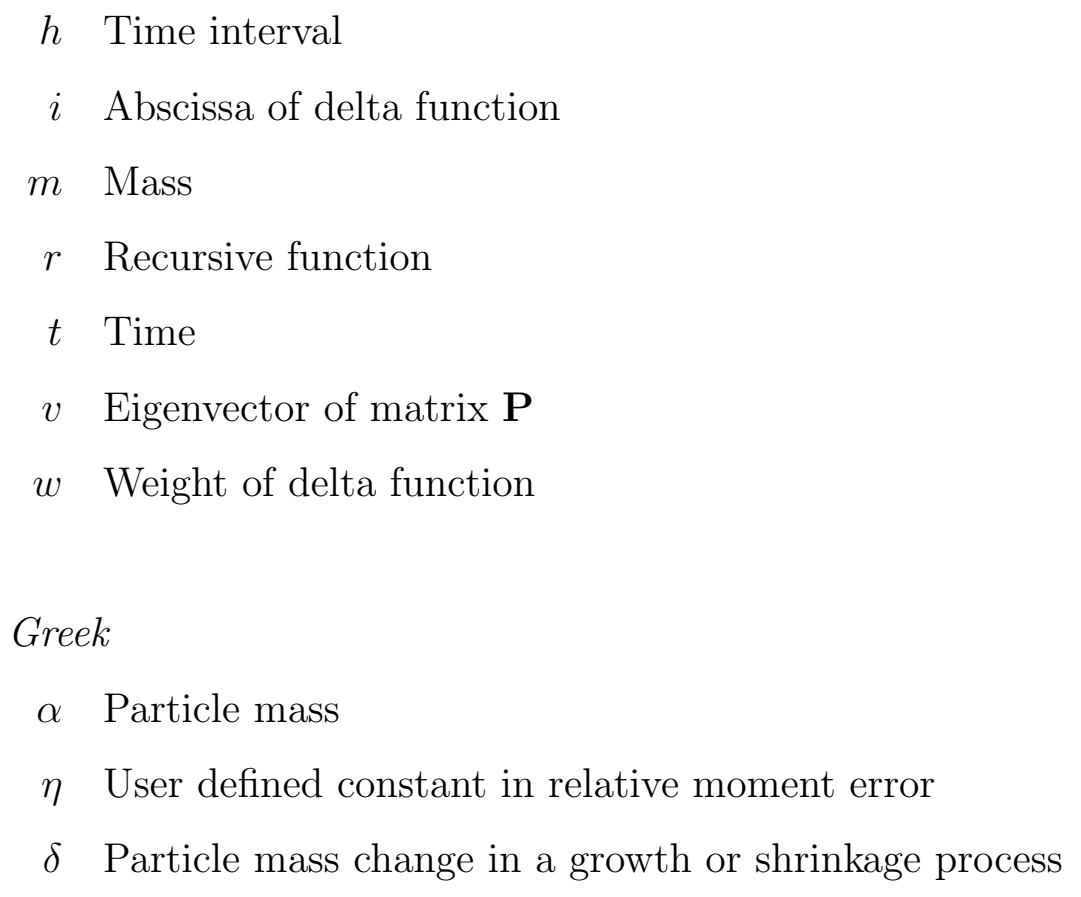

\section{Subscripts}

$\alpha$ Particle mass

f Final

L Large

$\max$ Maximum

p Particle

0 Initial or smallest

\section{Symbols}


$\widetilde{x} \quad$ Approximation of $x$

\section{Abbreviations}

DQMOM Direct quadrature method of moments

DSA Direct simulation algorithm

EQMOM Extended quadrature method of moments

FCMOM Finite-size domain complete set of trial functions method of moments

${ }_{483}$ HMOM Hybrid method of moments

MOM Method of moments

MOMIC Method of moments with interpolative closure

MPM Moment projection method

ODE Ordinary differential equation

PBE Population balance equation

PD Product difference

PSD Particle size distribution

QMOM Quadrature method of moments

\section{Appendix A. Blumstein-Wheeler algorithm}

This algorithm is used to determine the particle masses and the numbers used to approximate the PSD from the empirical moments. The algorithm is implemented in Matlab and makes use of the eig function to determine the eigenvalues and eigenvectors. 
Algorithm 2: Blumstein-Wheeler algorithm.

Input: The empirical moments $\widetilde{M}_{k}$ for $k=0,1, \ldots, 2 N_{\mathrm{p}}-2$.

Output: The particle masses $\alpha_{j}$ and the corresponding number of particles $\widetilde{N}_{\alpha_{j}}$ for

$$
j=1,2, \ldots, N_{\mathrm{p}} .
$$

Create a $N_{\mathrm{p}} \times 2 N_{\mathrm{p}}$ matrix $\mathbf{Z}$ with zeros in all elements.

Determine the elements of the first row of matrix $\mathbf{Z}: Z_{1, l}=\widetilde{M}_{l-1}$ for $l=1, \ldots, 2 N_{\mathrm{p}}-1$.

For $a_{1}=\widetilde{M}_{1} / \widetilde{M}_{0}$ and $b_{1}=0$, determine the recursion coefficients $a_{k}$ and $b_{k}$ :

for $k=2$ to $N_{p}$ do

for $l=k$ to $2 N_{p}-1$ do

The elements of $\mathbf{Z}$ must satisfy the following recursion relation:

$$
\begin{array}{r}
Z_{k, l}=Z_{k-1, l+1}-a_{k-1} Z_{k-1, l}-b_{k-1} Z_{k-1, l} ; \\
a_{k}=\frac{Z_{k, k+1}}{Z_{k, k}}-\frac{Z_{k-1, k}}{Z_{k-1, k-1}} ; \quad b_{k}=\frac{Z_{k, k}}{Z_{k-1, k-1}} .
\end{array}
$$

For $r_{1}=1 /\left(m_{1}-a_{1}\right)$ where $m_{1}$ is the smallest particle mass, determine the recursion function:

$$
r_{k}=1 /\left(m_{1}-a_{k}-b_{k} r_{k-1}\right) \quad k=2, \ldots, N_{\mathrm{p}}-1 .
$$

As we fix the smallest particle mass, replace $a_{N_{\mathrm{p}}}$ with:

$$
a_{N_{\mathrm{p}}}=m_{1}-b_{N_{\mathrm{p}}} r_{N_{\mathrm{p}}-1}
$$

Construct a symmetric tridiagonal matrix $\mathbf{P}$ with $a_{k}$ as the diagonal and the square roots of $b_{k}$ as the co-diagonal:

$$
\mathbf{P}=\left[\begin{array}{ccccc}
a_{1} & -\sqrt{b_{2}} & 0 & \cdots & 0 \\
-\sqrt{b_{2}} & a_{2} & -\sqrt{b_{3}} & \ldots & 0 \\
0 & -\sqrt{b_{3}} & a_{3} & \cdots & 0 \\
\vdots & \vdots & \vdots & \ddots & \vdots \\
0 & 0 & 0 & \cdots & a_{\mathrm{N}_{p}}
\end{array}\right]
$$

Solve for the eigenvalues $\mathbf{V}$ and eigenvectors $\mathbf{D}$ of matrix $\mathbf{P}$ :

$$
[\mathbf{V}, \mathbf{D}]=\operatorname{eig}(\mathbf{P})
$$

Solve for $\alpha_{j}$ and $\widetilde{N}_{\alpha_{j}}$ :

$$
\alpha_{j}=\mathbf{V}(j, j), \quad \widetilde{N}_{\alpha_{j}}=\widetilde{M}_{0} \mathbf{D}(1, j)^{2}
$$




\section{Appendix B. Hybrid method of moments}

HMOM was originally developed for bivariate PBEs based on particle volume and surface area $[39,44]$. Here we revise the method to be based on particle mass and we focus on the shrinkage process. Particles are discretised into two modes: particles of the smallest mass $i_{0}$ and particles of large mass $i_{\mathrm{L}}[39,44]$. Based on this concept, the $k$-th order moment is:

$$
M_{k}=N_{i_{0}} i_{0}^{k}+N_{i_{\mathrm{L}}} i_{\mathrm{L}}^{k}
$$

where $N_{i_{0}}$ and $N_{i_{\mathrm{L}}}$ are the number of particles of mass $i_{0}$ and $i_{\mathrm{L}}$, respectively. Combining Eqs. (2) and (17), we get:

$$
\frac{\mathrm{d} M_{k}}{\mathrm{~d} t}=-K_{\mathrm{Sk}} i_{0}^{k} N_{i_{0}}+K_{\mathrm{Sk}} \sum_{i=i_{0}+\delta}^{\infty}\left((i-\delta)^{k}-i^{k}\right) N_{i},
$$

where $K_{\mathrm{Sk}}$ is the shrinkage kernel and $\delta$ is the change in mass after a shrinkage process. The first term corresponds to the removal of the smallest particles when they shrink and the second term corresponds to the formation of the smallest particles when large particles shrink. Combining Eqs. (B.1) and (B.2):

$$
\frac{\mathrm{d} M_{k}}{\mathrm{~d} t}= \begin{cases}-K_{\mathrm{Sk}} N_{i_{0}}, & k=0 \\
K_{\mathrm{Sk}} \sum_{r=1}^{k}\left(\begin{array}{l}
k \\
r
\end{array}\right)(-\delta)^{r}\left(i_{0}^{k-r} N_{i_{0}}+i_{\mathrm{L}}^{k-r} N_{i_{\mathrm{L}}}\right), & k>0 .\end{cases}
$$

The source term for $N_{i_{0}}$ is given by ref. [39]:

$$
\frac{\mathrm{d} N_{i_{0}}}{\mathrm{~d} t}=\lim _{k \rightarrow-\infty} \frac{\mathrm{d} M_{k} / \mathrm{d} t}{i_{0}^{k}} .
$$

4 Applying Eq. (B.4) to Eq. (B.2) we get:

$$
\frac{\mathrm{d} N_{i_{0}}}{\mathrm{~d} t}=-K_{\mathrm{Sk}} N_{i_{0}}+K_{\mathrm{Sk}} N_{i_{0}+\delta} .
$$


The first term is the destruction of the smallest particles and the second term corresponds to the intermodal transfer of particles from the second mode to the first during a shrinkage process. To close this latter term, in ref. [44] it is assumed that the number of particles transferred from the large particles to the smallest particles is proportional to the total mass lost from the large particles with a coefficient, $C$, equal to the mass ratio between the two modes $i_{0} / i_{\mathrm{L}}:$

$$
N_{i_{0}+\delta}=C \delta M_{-1}^{\mathrm{L}}=\frac{i_{0} \delta}{i_{\mathrm{L}}^{2}} N_{i_{\mathrm{L}}},
$$

where the superscript L refers to the contribution to the moment from the second mode. Combining Eqs. (B.5) and (B.6):

$$
\frac{\mathrm{d} N_{i_{0}}}{\mathrm{~d} t}=-K_{\mathrm{Sk}} N_{i_{0}}+\frac{i_{0} \delta}{i_{\mathrm{L}}^{2}} K_{\mathrm{Sk}} N_{i_{\mathrm{L}}}
$$

The remaining two quantities in Eq. (B.3) are obtained from the two known moments [44]:

$$
N_{i_{\mathbf{L}}}=M_{0}-N_{i_{0}}
$$

and

$$
i_{\mathrm{L}}=\frac{M_{1}-N_{i_{0}} i_{0}}{N_{i_{\mathrm{L}}}} .
$$

517 Algorithm 3 describes the numerical procedure of HMOM for the shrinkage process. The HMOM approach for other processes (inception, coagulation and growth) can be obtained in a similar way, but the details are not given here for simplicity. 


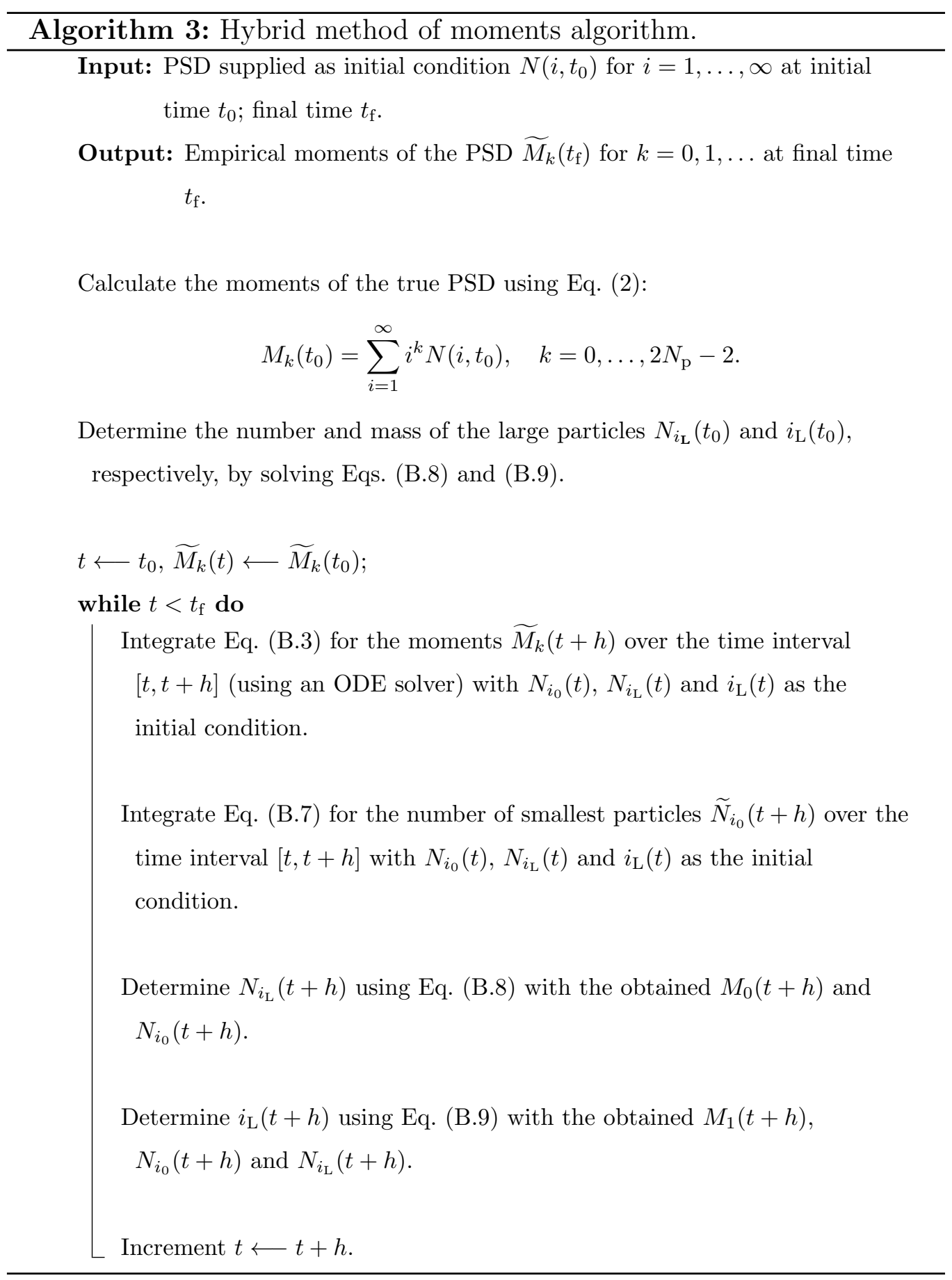


[1] S. Rigopoulos, Population balance modelling of polydispersed particles in reactive flows, Prog. Energ. Combust. 36 (2010) 412-443. doi:10.1016/j.pecs.2009.12.001.

[2] A. D. Randolph, M. A. Larson, Theory of Particulate Processes: Analysis and Techniques of Continuous Crystallization (second ed.), Academic Press, San Diego, 1988.

[3] D. Ramkrishna, Population Balances: Theory and Applications to Particulate Systems in Engineering, Academic Press, New York, 2000.

[4] S. Shekar, A. J. Smith, W. J. Menz, M. Sander, M. Kraft, A multidimensional population balance model to describe the aerosol synthesis of silica nanoparticles, J. Aerosol Sci. 44 (2012) 83-98. doi:10.1016/j.jaerosci.2011.09.004.

[5] S. Shekar, W. J. Menz, A. J. Smith, M. Kraft, W. Wagner, On a multivariate population balance model to describe the structure and composition of silica nanoparticles, Comput. Chem. Eng. 43 (2012) 130-147. doi:10.1016/j.compchemeng.2012.04.010.

[6] J. Akroyd, A. J. Smith, R. Shirley, L. R. McGlashan, M. Kraft, A coupled CFD-population balance approach for nanoparticle synthesis in turbulent reacting flows, Chem. Eng. Sci. 66 (2011) 3792-3805. doi:10.1016/j.ces.2011.05.006.

[7] E. Madadi-Kandjani, A. Passalacqua, An extended quadrature-based moment method with log-normal kernel density functions, Chem. Eng. Sci. 131 (2015) 323-339. doi:10.1016/j.ces.2015.04.005. 
[8] R. I. A. Patterson, W. Wagner, M. Kraft, Stochastic weighted particle methods for population balance equations, J. Comput. Phys. 230 (2011) 7456-7472. doi:10.1016/j.jcp.2011.06.011.

[9] M. Kraft, W. Wagner, Numerical study of a stochastic particle method for homogeneous gas-phase reactions, Comput. Math. Appl. 45 (2003) 329-349. doi:10.1016/S0898-1221(03)80022-6.

[10] D. Grosschmidt, H. Bockhorn, M. Goodson, M. Kraft, Two approaches to the simulation of silica particle synthesis, Proc. Combust. Inst. 29 (2002) 1039-1046. doi:10.1016/S1540-7489(02)80131-6.

[11] M. Balthasar, M. Kraft, A stochastic approach to calculate the particle size distribution function of soot particles in laminar premixed flames, Combust. Flame. 133 (2003) 289-298. doi:10.1016/S00102180(03)00003-8.

[12] A. Vikhansky, M. Kraft, Single-particle method for stochastic simulation of coagulation processes, Chem. Eng. Sci. 60 (2005) 963-967. doi:10.1016/j.ces.2004.09.062.

[13] A. Eibeck, W. Wagner, Stochastic interacting particle systems and nonlinear kinetic equations, Ann. Appl. Probab. 13 (2003) 845-889. doi:10.1214/aoap/1060202829.

[14] A. Braumann, M. Kraft, W. Wagner, Numerical study of a stochastic particle algorithm solving a multidimensional population balance model for high shear granulation, J. Comput. Phys. 229 (2010) 7672-7691. doi:10.1016/j.jcp.2010.06.021. 
[15] E. K. Y. Yapp, R. I. A. Patterson, J. Akroyd, S. Mosbach, E. M. Adkins, J. Houston Miller, M. Kraft, Numerical simulation and parametric sensitivity study of optical band gap in a laminar coflow ethylene diffusion flame, Combust. Flame 167 (2016) 320-334. doi:10.1016/j.combustflame.2016.01.033.

[16] S. Kumar, D. Ramkrishna, On the solution of population balance equations by discretization-I. A fixed pivot technique, Chem. Eng. Sci. 51 (1996) 1311-1332. doi:10.1016/0009-2509(96)88489-2.

[17] F. Gelbard, Y. Tambour, J. H. Seinfeld, Sectional representations for simulating aerosol dynamics, J. Colloid Interface Sci. 76 (1980) 541556. doi:10.1016/0021-9797(80)90394-X.

[18] M. D. Smooke, C. S. McEnally, L. D. Pfefferle, R. J. Hall, M. B. Colket, Computational and experimental study of soot formation in a coflow, laminar diffusion flame, Combust. Flame. 117 (1999) 117-139. doi:10.1016/S0010-2180(98)00096-0.

[19] S. Kumar, D. Ramkrishna, On the solution of population balance equations by discretization-II. A moving pivot technique., Chem. Eng. Sci. 51 (1996) 1333-1342. doi:10.1016/0009-2509(95)00355-X.

[20] M. C. Bruns, O. A. Ezekoye, Development of a hybrid sectional quadrature-based moment method for solving population balance equations, J. Aerosol Sci. 54 (2012) 88-102. doi:10.1016/j.jaerosci.2012.07.003. 
[21] M. J. Hounslow, R. L. Ryall, V. R. Marshall, Discretized population balance for nucleation, growth and aggregation, AIChE J. 34 (1988) 1821-1832. doi:10.1002/aic.690341108.

[22] M. J. Hounslow, A discretized population balance for continuous systems at steady state, AIChE J. 36 (1990) 106-116. doi:10.1002/aic.690360113.

[23] V. Alopaeus, M. Laakkonen, J. Aittamaa, Solution of population balances with breakage and agglomeration by high-order momentconserving method of classes, Chem. Eng. Sci. 61 (2006) 6732-6752. doi:10.1016/j.ces.2006.07.010.

[24] D. L. Marchisio, R. D. Vigil, R. O. Fox, Quadrature method of moments for aggregation-breakage processes, J. Colloid Interface Sci. 258 (2003) 322-334. doi:10.1016/S0021-9797(02)00054-1.

[25] H. M. Hulburt, S. Katz, Some problems in particle technology: a statistical mechanical formulation, Chem. Eng. Sci. 19 (1964) 555-574. doi:10.1016/0009-2509(64)85047-8.

[26] J. C. Barrett, N. A. Webb, A comparison of some approximate methods for solving the aerosol general dynamic equation, J. Aerosol Sci. 29 (1998) 31-39. doi:10.1016/S0021-8502(97)00455-2.

[27] J. Singh, M. Balthasar, M. Kraft, W. Wagner, Stochastic modeling of soot particle size and age distributions in laminar premixed flames, Proc. Combust. Inst. 30 (2005) 1457-1465. doi:10.1016/j.proci.2004.08.120. 
[28] J. Akroyd, A. J. Smith, L. R. McGlashan, M. Kraft, Numerical investigation of DQMoM-IEM as a turbulent reaction closure, Chem. Eng. Sci. 65 (2010) 1915-1924. doi:10.1016/j.ces.2009.11.010.

[29] J. Akroyd, A. J. Smith, L. R. McGlashan, M. Kraft, Comparison of the stochastic fields method and DQMoM-IEM as turbulent reaction closures, Chem. Eng. Sci. 65 (2010) 5429-5441. doi:10.1016/j.ces.2010.06.039.

[30] M. Frenklach, Method of moments with interpolative closure, Chem. Eng. Sci. 57 (2002) 2229-2239. doi:10.1016/S0009-2509(02)00113-6.

[31] M. Frenklach, S. J. Harris, Aerosol dynamics modeling using the method of moments, J. Colloid Interface Sci. 118 (1987) 252-261. doi:10.1016/0021-9797(87)90454-1.

[32] R. McGraw, Description of aerosol dynamics by the quadrature method of moments, Aerosol Sci. Technol. 27 (1997) 255-265. doi:10.1080/02786829708965471.

[33] D. L. Marchisio, J. T. Pikturna, R. O. Fox, R. D. Vigil, A. A. Barresi, Quadrature method of moments for population-balance equations, AIChE. J. 49 (2003) 1266-1276. doi:10.1002/aic.690490517.

[34] D. L. Marchisio, R. O. Fox, Solution of population balance equations using the direct quadrature method of moments, J. Aerosol Sci. 36 (2005) 43-73. doi:10.1016/j.jaerosci.2004.07.009.

[35] L. F. L. R. Silva, R. C. Rodrigues, J. F. Mitre, P. L. C. Lage, Comparison of the accuracy and performance of quadrature-based 
methods for population balance problems with simultaneous breakage and aggregation, Comput. Chem. Eng. 34 (2010) 286-297. doi:10.1016/j.compchemeng.2009.11.005.

[36] M. Kraft, Modelling of particulate processes, KONA Powder Part. J. 23 (2005) 18-35. doi:10.14356/kona.2005007.

[37] M. Strumendo, H. Arastoopour, Solution of PBE by MOM in finite size domains, Chem. Eng. Sci. 63 (2008) 2624-2640. doi:10.1016/j.ces.2008.02.010.

[38] C. Yuan, F. Laurent, R. O. Fox, An extended quadrature method of moments for population balance equations, J. Aerosol Sci. 51 (2012) 1-23. doi:10.1016/j.jaerosci.2012.04.003.

[39] M. E. Mueller, G. Blanquart, H. Pitsch, Modeling the oxidation-induced fragmentation of soot aggregates in laminar flames, Proc. Combust. Inst. 33 (2011) 667-674. doi:10.1016/j.proci.2010.06.036.

[40] R. G. Gordon, Error bounds in equilibrium statistical mechanics, J. Math. Phys. 9 (1968) 655-663. doi:10.1063/1.1664624.

[41] C. Blumstein, J. C. Wheeler, Modified-moments method: applications to harmonic solids, Phys. Rev. B 8 (1973) 1764-1776. doi:10.1103/PhysRevB.8.1764.

[42] W. B. Gragg, Matrix interpretations and applications of the continued fraction algorithm, Rocky Mountain J. Math. 4 (1974) 213-226. doi:10.1216/RMJ-1974-4-2-213. 
[43] J. C. Wheeler, Modified moments and Gaussian quadratures, Rocky Mountain J. Math. 4 (1974) 287-296. doi:10.1216/RMJ-1974-4-2-287.

[44] M. E. Mueller, G. Blanquart, H. Pitsch, Hybrid method of moments for modelling soot formation and growth, Combust. Flame. 156 (2009) 1143-1155. doi:10.1016/j.combustflame.2009.01.025. 